\title{
The onset of solar energetic particle events: prompt release of deka-MeV protons and associated coronal activity $\star$
}

\author{
K.-L. Klein ${ }^{1}$ and A. Posner ${ }^{2}$ \\ 1 Observatoire de Paris, LESIA-CNRS UMR 8109, 92195 Meudon, France \\ e-mail: ludwig.klein@obspm. fr \\ 2 Southwest Research Institute, Space Science and Engineering Division, 6220 Culebra Rd., San Antonio, TX 78238, USA \\ e-mail: aposner@swri.org
}

Received 23 December 2004 / Accepted 7 March 2005

\begin{abstract}
Between 1996 and 2002, the COSTEP particle instrument aboard the SoHO mission observed 18 solar energetic particle events where the release times of protons up to $54 \mathrm{MeV}$ could be determined to within an uncertainty of about ten minutes, and where metre wave imaging observations with the Nançay Radioheliograph were available. In six of them the proton release starts, within the uncertainty of the method, simultaneously with the release of electron beams observed through their type III emission at decametric-to-kilometric wavelengths, during flares in $\mathrm{H} \alpha$ or EUV. These well-connected events are studied to identify the coronal processes that occur when the particle release starts at the Sun. Given that big events are not reliably measured by COSTEP due to its large geometric factor, they are rather weak with peak intensities $\left(9 \times 10^{-4}-5 \times 10^{-2}\right)\left[\left(\mathrm{cm}^{2} \mathrm{~s} \mathrm{sr} \mathrm{MeV}\right)^{-1}\right]$ in the (8-25) MeV range. All are accompanied by fast (400-1300 $\mathrm{km} \mathrm{s}^{-1}$ ) and narrow coronal mass ejections (CMEs). While half of the events is accompanied by significant microwave bursts, the other half shows little or no evidence of electron acceleration in the low corona and rather weak soft X-ray bursts. The association with weak flares may point to different acceleration sites of the interacting and the escaping particles. From the radio observations of the type III bursts and the associated metre wave emission, the escaping electrons, and by inference also the first escaping protons, are accelerated roughly between $0.1 R_{\odot}$ and $0.5 R_{\odot}$ above the photosphere, a height range shown to be well behind the front of the CMEs at the time of acceleration. The data show no evidence that the shock waves, which are presumably driven by at least the fastest of these CMEs, participate in the early acceleration of the escaping protons or affect the escape of the protons accelerated at lower height.
\end{abstract}

Key words. Sun: coronal mass ejections (CMEs) - Sun: flares - Sun: particle emission - Sun: radio radiation

\section{Introduction}

Transient enhancements of energetic particle fluxes in space, termed solar energetic particle events (henceforth SEP events), are related to both solar flares and coronal mass ejections (CMEs). Based on time profiles, charge states, and composition measured near $1 \mathrm{AU}$ up to $\mathrm{MeV}$-energies, it has become general use (Reames 1999) to discuss their origin in terms of two distinct mechanisms: large particle events are ascribed to acceleration at the bow shocks of fast coronal mass ejections, while the numerous small low-energy events are thought to arise during magnetic reconnection in flaring active regions. Few studies have sought to disentangle the complex, but nearly always encountered situation where both a flare and a CME are observed near the onset of an SEP event. The conclusion that no "flare-accelerated" particles contribute to large SEP events relies on the belief that particles accelerated in the low and middle corona are prevented from travelling to Earth either by the bow shock of the CME (Kahler 1996) or because they

* Appendix A is only available in electronic form at http://www. edpsciences.org are supposedly trapped in the coronal magnetic fields (Reames 2002). Both claims, as plausible as they may appear, have no direct observational justification. Yet, in light of the new observations of time-and energy-dependent elemental compositions and charge states (Möbius et al. 2002, 2003) it is important to know if particles from the low and the middle corona contribute to CME-associated SEP events or not. The answer may be energy-dependent; e.g., shock acceleration may be the most appealing mechanism for particles at and below $\mathrm{MeV}$-energies, but not for relativistic protons (cf. discussion in Klein \& Trottet 2001). The present work addresses the early release of protons at energies up to $54 \mathrm{MeV}$, which we refer to as "deka-MeV protons".

The contribution of coronal flare-like acceleration to large particle events is supported by the statistical association, within a few hours, of the onset of SEP events at tens of MeV per nucleon with $\mathrm{keV}$ electron beams emanating from the corona and traced by their decametric-to-hectometric (DH in the following) type III radio emission (Cane et al. 2002). In the present analysis we use a more precise determination of the initial release time of deka-MeV protons, based on the dispersion 
of particle arrival times at the Comprehensive Suprathermal and Energetic Particle Analyser (COSTEP) instrument aboard the Solar and Heliospheric Observatory (SoHO). We focus on well-connected events where the proton release occurs together with DH type III bursts and flares in the western solar hemisphere, and investigate the coronal counterpart of the type III bursts within $0.5 R_{\odot}$ above the photosphere by metre-wave imaging observations with the Nançay Radioheliograph. We also use context observations of coronal plasma structures from the SoHO and Yohkoh spacecraft. The instrumentation and the technique of release time determination of protons are discussed in Sect. 2. Section 3 gives an overview of results for the six events between 1996 and 2002, as well as two detailed examples. The others are presented in the Appendix. Implications of these observations are discussed in Sect. 4.

\section{Observations}

\subsection{Instruments and data analysis}

The strategy of the present investigation is to derive the times when energetic protons start to be released at the Sun, using the arrival of the first protons at the spacecraft at $1 \mathrm{AU}$, and to study radio signatures of non-thermal electrons in the corona as the tracers of local acceleration processes at the time of the initial proton release. Context observations of the coronal plasma and its variations are employed to identify the regions where the coronal electron acceleration occurs.

\subsubsection{COSTEP measurements of deka-MeV protons}

The COSTEP instrument (Müller-Mellin et al. 1995) aboard SoHO is designed for the observation of the energetic particle environment at $1 \mathrm{AU}$. The COSTEP/EPHIN sensor has the ability to detect solar energetic particle events with intermediate to high intensities ranging from $10^{-5}$ up to $10^{3}\left(\mathrm{~cm}^{2} \mathrm{sr} \mathrm{s} \mathrm{MeV}\right)^{-1}$. This includes ions in the range $\sim 4$ to $54 \mathrm{MeV} / \mathrm{amu}$ and electrons between $200 \mathrm{keV}$ and $11 \mathrm{MeV}$. COSTEP/EPHIN consists of a stack of 6 solid state detectors (SSDs) within active anticoincidence shielding.

The COSTEP/EPHIN data products are twofold. The fast onboard classification scheme uses predefined energy channels for electrons, protons, and helium. Count rates are generated using all of the above particles measured within the accumulation interval of one minute. However, a more thorough treatment of the onboard fluxes is sometimes necessary in order to take instrumental effects such as crosstalk into account, but also to distinguish isotopes of hydrogen and helium, which is not achieved with onboard processing. For this purpose, pulseheight analysed (PHA) data is produced that contains energy losses of individual particles in the SSDs. For low-flux periods, all detected particles are pulse-height analyzed. However, a priority buffer scheme is applied if the allocated telemetry for PHA data is reached in an accumulation interval. This scheme favors rare species (such as $\mathrm{p}, \mathrm{He}$ ) over more abundant species (such as electrons) in order to guarantee a minimum statistical sample for the correction of the energy channels with count rates. Within each channel, the PHA words have the same level of priority. All scientific data products of COSTEP/EPHIN incorporate the use of this weighting scheme, along with $\mathrm{d} E / \mathrm{d} x$ vs. $E$ filtering (McDonald \& Ludwig 1964) and deadtime correction.

\subsubsection{Radio observations at wavelengths $>50 \mathrm{~cm}$ : suprathermal electrons from $0.1 R_{\odot}$ to $1 \mathrm{AU}$}

The proton release time is compared to different signatures of energetic electrons in the corona and the solar wind. 1-min integrated spectra of the WAVES experiment on the Wind spacecraft (Bougeret et al. 1995), provided via the instrument homepage at NASA Goddard Space Flight Center (GSFC) ${ }^{1}$, were used to monitor radio bursts in the high corona and interplanetary space. This instrument provides dynamic spectra of the solar radio emission between $14 \mathrm{MHz}$ and $4 \mathrm{kHz}$. Electron beams travelling outward through the corona along open magnetic flux tubes are revealed by their type III emission, which is electromagnetic emission produced through the conversion of Langmuir waves that the electron beams excite at ever lower frequencies while travelling outward. The frequency of the radio emission is near either the local plasma frequency or its harmonic, depending on the process by which the energy is transferred from the Langmuir waves to the radio waves. Type II bursts are generated in a similar way, but by electrons accelerated at a coronal shock wave. The emission also drifts to lower frequencies, but at a slower rate that reflects the slower speed of the shock as compared to the type III emitting electron beams. The frequency range of the WAVES spectrograph corresponds to heliocentric distances between roughly $3 R_{\odot}$ and $1 \mathrm{AU}$. Spectral observations at higher frequencies are provided by ground-based observatories. We use data reports from the instruments of IZMIRAN Moscow, the Nançay Decametric Array (NDA; Lecacheux 2000), the Potsdam-Tremsdorf Observatory (Mann et al. 1992) and Zurich through their web-based reports ${ }^{2}$ or those published in Solar Geophysical Data (henceforth abbreviated as $S G D$ ). These instruments cover frequency ranges from the base of the corona to a heliocentric distance of about $2 R_{\odot}$. At long decimetric and metric wavelengths the emitting sources can be localised by the Nançay Radioheliograph (NRH in the following, Kerdraon \& Delouis 1997). It consists of a T-shaped array of 44 antennas. It maps radio emission routinely at five frequencies between 164 and $432 \mathrm{MHz}$, with spatial resolution of a few arcminutes at $164 \mathrm{MHz}$ and sub-second time resolution. Data integrated over 10 or $30 \mathrm{~s}$ were used in this study, unless stated otherwise.

\subsubsection{Spectrography at $\mathrm{cm}$-wavelengths: mildly relativistic electrons in the low corona}

While the decimetric-to-kilometric radio observations are a good tracer of electron beams and shock waves from the corona

\footnotetext{
${ }^{1}$ http://lep694.gsfc.nasa.gov/waves/waves.html

2 http://helios.izmiran.rssi.ru/lars/LARS.html, http://www. obs-nancay.fr, http://www .aip.de/groups/osra/, http://www . astro.phys.ethz.ch/rapp/
} 

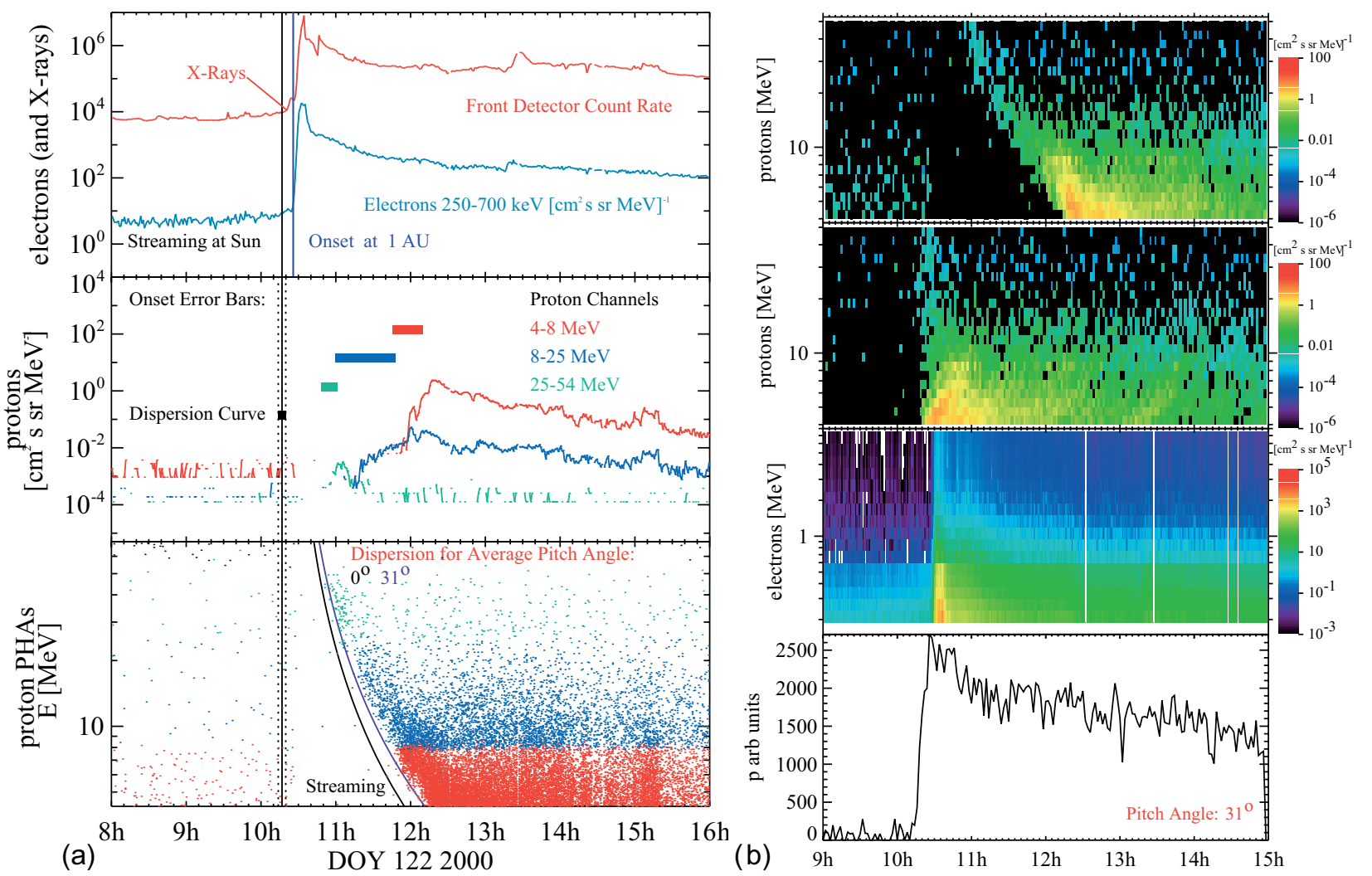

Fig. 1. Eight hours of SOHO/COSTEP electron and proton observations on 2000 May 01. a) The top panel shows 250-700 keV electron intensities (blue) along with the EPHIN front detector count rate (red) that is dominated by electrons, but also sensitive to X-rays. The second panel shows intensities of the available proton channels from 4 to $54 \mathrm{MeV}$. Coloured horizontal bars indicate the dependence of the onset uncertainty for the given channels on the energy width stemming from the velocity dispersion. The bottom panel represents individual pulse height analysed protons over time, and the colours refer to channels of the middle panel. The curved lines in the bottom panel give the fitted onset time using the nominal Parker spiral length and average pitch angles of $0^{\circ}$ and $31^{\circ}$, respectively. The solid vertical line marks the inferred proton release time at the Sun, the dashed lines and the black rectangle its statistical uncertainty. b) Particle intensities in the energy-time plane. From top to bottom: protons (1; same as in the bottom panel of a)), (2) onset-time aligned protons after the shift by the travel time inferred from the onset time analysis, (3) electrons, (4) count rate time history of protons after aligning the different onset times.

to interplanetary space, the best understood diagnostics of energetic electrons in the low corona are their hard X-ray and microwave (i.e. radio at frequencies above $1 \mathrm{GHz}$ ) emission. In this study we use the time histories of whole Sun microwave flux densities at fixed frequencies (notably 2.695, 4.995, 8.800, and $15.4 \mathrm{GHz}$ ) from the Sagamore Hill radio observatory, kindly provided through the RSTN network and the National Oceanic and Atmospheric Administration (NOAA) at Boulder and from the University of Bern. In the RSTN data of 1999 and 2000 the time axis had to be corrected by comparison of the simultaneously provided flux densities at 245 and $410 \mathrm{MHz}$ to NRH observations at neighbouring frequencies.

\subsubsection{Context observations of the coronal plasma}

Besides using the microwave emission, which in general refers to nonthermal electrons, energy release in the corona is traced by whole Sun soft X-ray monitoring of the thermal plasma by the Geostationary Operational Environmental Satellites (GOES, Garcia 1994). Data with 3 s time resolution are provided by the Solar Data
Analysis Center (SDAC) at NASA-GSFC ${ }^{3}$. In order to identify flares and mass ejections, context observations of the solar corona from the Extreme Ultraviolet Imaging Telescope (EIT, Delaboudinière et al. 1995) and the Large Angle Spectroscopic Coronographs (LASCO, Brueckner et al. 1995) on the SoHO spacecraft and images from the Soft X-ray Telescope aboard the Yohkoh mission (Tsuneta et al. 1991) were used. Parameters of coronal mass ejections were drawn from the CME catalogue maintained by S. Yashiro at the Catholic University of America and NASA-GSFC (http://cdaw.gsfc.nasa.gov/CME_list) and from the daily LASCO movies provided by the web-pages of the Naval Research Laboratory, Washington, and the Max-Planck-Institut für Sonnensystemforschung, Lindau ${ }^{4}$. EIT data was provided by the SOHO archive web-site ${ }^{5}$. The listings of $\mathrm{H} \alpha$ flares in $S G D$ were also used to identify flare sites in the chromosphere.

\footnotetext{
${ }^{3}$ http://umbra.nascom.nasa.gov

${ }^{4}$ http://lasco-www.nrl.navy.mil/lasco.html,

http://star.mpae.gwdg.de

${ }^{5}$ http://sohowww.estec.esa.nl
} 


\subsection{Timing of proton release}

We display PHA data of protons over time to analyse the onset of solar energetic particle events, where velocity dispersion signifies systematically the early arrival of high energy (velocity) particles of one species over the later arrival at lower energies (velocities). Under the assumption that all particles are released at the same time and place, this propagation effect can be used to infer the travel distance of these particles.

As an example, Fig. 1a shows electron and proton fluxes (two top panels) and proton PHA data over time (bottom panel) for the SEP event of 2000 May 01. In the top panel we included the COSTEP/EPHIN front detector count rate (cts/s). Usually, this channel is dominated by $200-700 \mathrm{keV}$ electrons, which are also shown in a separate curve. Excess counts in the front detector count rate are due to the frequent occurrence of solar X-ray events at about the time of the onset of SEP events. Only a weak signal is observed in this example. All electron channels of COSTEP/EPHIN are in the relativistic energy range with expected arrival time differences of minutes, which are comparable to the temporal resolution of the instrument. Relativistic electron onsets therefore are not analysed in detail, but they can be used as a timer for the release of ions, provided that electrons and ions are released at the same time.

Energy dispersion can be recognised in the rises of ion intensity profiles in different energy channels, as shown for protons in the middle panel. The accuracy of determining the solar release time from the onset of energy channels is limited by the width of the energy range, which directly translates into a temporal uncertainity; e.g., the onset of the (8-25) MeV proton channel (blue) spans over $45 \mathrm{~min}$. The uncertainity of this method can only be reduced by assumptions. In order to effectively reduce the uncertainity of particle release time from this effect alone, it is necessary to know the high-energy cutoff of these protons, since the highest energy particles of a given channel arrive first and most likely raise the flux above the background level.

In this paper, we use PHA data for onset time analysis, which allows us to define custom-made energy steps that can be used for analysis as compared to integrating over channels of a predefined, fixed energy width. The bottom panel of Fig. 1a displays proton PHAs in an energy-over-time representation. In this representation, even weak events can be recognised by the velocity dispersion pattern that cannot otherwise be deciphered out of time profiles of energy channels alone. With high accuracy ( \pm 3 min, black bar in Panel 2$)$, the starting point of the streaming of energetic ions can be determined, as compared to methods using onsets within pre-defined energy channels (uncertainity on the order of $>10$ min indicated by coloured horiziontal bars in panel 2). When the proton arrival at the spacecraft was uncertain because of confusion with a previous event, He ions were used.

The technique to derive the solar release time from COSTEP/EPHIN particle data is based on the $1 / \beta$ method under the assumption that ions with energies (4-54) $\mathrm{MeV} /$ nuc are released simultaneously. The resulting energy dispersion of the arrival time at the spacecraft then depends on the distance along the interplanetary magnetic field $(S)$ and on pitch angle $(\alpha)$ according to the relation:

$t=\frac{S\left(V_{\mathrm{sw}}\right)}{V_{\mathrm{part}} \cos \alpha}$

where $V_{\text {part }}$ and $V_{\text {sw }}$ represent the speeds of the particle and of the solar wind, respectively.

The distance to the Sun along the magnetic field was derived from the Archimedean spiral equation with a radial distance of $1 \mathrm{AU}$ and an opening angle that is defined by the Sun's angular rotation speed and the in situ solar wind speed. Note that local magnetic field irregularities tend to increase the distance to the Sun as derived with this method. The pitch angle used here therefore should be understood as a characteristic pitch angle for the first arriving particles. For comparison the bottom panel of Fig. 1a shows results of onset fits to the PHA data for the cases of $0^{\circ}$ and $31^{\circ}$ pitch angle. The fitting method is outlined below.

In Fig. 1b, we transformed PHA data and energy channel information into energetic particle spectrograms. The time resolution for proton spectrograms is $2 \mathrm{~min}$, for electrons $1 \mathrm{~min}$. The resulting energy bins are logarithmically spaced and colour coded according to the intensity scales given on the right. The top panel, again, shows protons.

Particle speed and energy are equivalent. Therefore, we assigned (average) speeds to the proton energy bins by taking any relativistic effects into account. The second and the bottom panels give the results for our onset fitting procedure. Equivalent to the commonly used $1 / \beta$ method, the individual energy bins are shifted by an assigned time that is equivalent to streaming at constant speed from a certain heliocentric distance, which is the only free parameter used. The fit is varied until the onset of the particle event lines up vertically over all energies, such as shown in the second panel (from top). As a reference, the bottom panel sums up the shifted flux values. The slope at the onset maximises when the fit is accomplished.

Note that the free parameter, the travel distance from the Sun, is usually longer than the connection distance along the ideal Parker spiral field line. This path length excess is treated here as the average pitch angle effect. In our example, the connection distance is $1.315 \mathrm{AU}$, while the spiral length is $1.1268 \mathrm{AU}$, therefore $\phi=31^{\circ}$. The solar release time (SRT) of protons coincides with the time of vertical alignment of the shifted proton onset in Panel 2. Note that for comparison with in situ electron onsets and with electromagnetic emissions from the Sun we use the time measured at Earth, which is equivalent to $\mathrm{SRT}+8 \mathrm{~min}$.

A check for our method is to consider the onset of the relativistic electrons, given in Panel 3 of Fig. 1b. According to our results here and elsewhere (Posner \& Hassler 2004), there is no reason to believe that electrons and ions are released at different times. However, previous authors (Neustock et al. 1985; Kallenrode \& Wibberenz 1991; Krucker \& Lin 2000; Tylka et al. 2003) do not agree with each other in this point. We find the arrival of electrons at the spacecraft approximately $10 \mathrm{~min}$ after the derived proton release time, as expected if both species are released at the same time. 
Table 1. Parameters of the energetic protons and the solar wind.

\begin{tabular}{|c|c|c|c|c|c|c|c|}
\hline \multirow[t]{3}{*}{ Date } & \multicolumn{4}{|c|}{ Protons } & \multicolumn{2}{|c|}{ Solar wind } & \multirow{3}{*}{$\begin{array}{r}\text { Flare } \\
\text { Longitude }\end{array}$} \\
\hline & $E_{\max }$ & $J_{\max }(8-25)$ & $J_{\max }(25-53)$ & Pitch & Speed & Longi- & \\
\hline & {$[\mathrm{MeV}]$} & \multicolumn{2}{|c|}{$\left[\left(\mathrm{cm}^{2} \mathrm{~s} \mathrm{sr} \mathrm{MeV}\right)^{-1}\right]$} & angle & {$\left[\mathrm{km} \mathrm{s}^{-1}\right]$} & tude & \\
\hline 20000501 & $>54$ & $5.29 \times 10^{-2}$ & $3.16 \times 10^{-3}$ & $31^{\circ}$ & 453 & $50^{\circ}$ & $51^{\circ}$ \\
\hline 19960709 & $>54$ & $8.35 \times 10^{-3}$ & $6.36 \times 10^{-4}$ & $35^{\circ}$ & 419 & $53^{\circ}$ & $30^{\circ}$ \\
\hline 20021020 & 30 & $<6.28 \times 10^{-3}$ & $<8.60 \times 10^{-4}$ & $52^{\circ}$ & 650 & $35^{\circ}$ & $18^{\circ}, 63^{\circ}$ \\
\hline 19990629 & $\sim 20$ & $3.59 \times 10^{-3}$ & $7.50 \times 10^{-4}$ & $20^{\circ}$ & 532 & $42^{\circ}$ & $45^{\circ}$ \\
\hline 20021212 & 25 & $<1.51 \times 10^{-3}$ & $1.26 \times 10^{-4}$ & $20^{\circ}$ & 362 & $63^{\circ}$ & $37^{\circ}$ \\
\hline 20021005 & 15 & $9.46 \times 10^{-4}$ & $<2.50 \times 10^{-4}$ & $0^{\circ}$ & (374) & $\left(61^{\circ}\right)$ & $63^{\circ}$ \\
\hline
\end{tabular}

Apparent injection delays due to particle interactions with the turbulent interplanetary magnetic field were discussed by Kallenrode \& Wibberenz (1990) and Lintunen \& Vainio (2004). Their numerical calculations show that the supplementary delay of proton event onsets can attain a significant fraction of the rectilinear travel time. As mentioned above, we have taken this at least partially into account by introducing an average pitch angle into the determination of travel time, instead of using the assumption that particles travel along the nominal interplanetary magnetic field at the speed implied by their energy.

\subsection{Event selection}

The event selection was largely determined by how accurately we were able to infer the onset time from COSTEP data. Accurate determination requires a good correspondence between event intensity and geometrical factor of the instrument. Relatively small events do not provide sufficient statistics for our method, while for very strong events the on-board electronics cannot handle the high frequency of incoming particles properly. Physical causes for sizable uncertainities are, e.g., high background fluxes from a previous injection. Heliospheric transport effects may cause slowly rising fluxes at 1 AU. Slowly rising injection profiles at the Sun may cause similar delays. The greater these effects, the larger the delay between the observed time when particle counts exceed the pre-event background and the true start of particle release; hence, the release time determination has a systematic error that will create apparent delays. Furthermore, the angular separation of the magnetic foot point of the interplanetary field line from the flare location may increase onset uncertainity due to effects such as cross field diffusion. Alternative physical explanations are the propagation of an accelerator (shock) across field lines or the propagation of a disturbance that allows release of particles. Hucke et al. (1992) and Kallenrode (1993) have shown with Helios observations that the intensity of SEP events rises more slowly with increasing azimuthal distance, which may lead to inferring a delayed onset. This is in agreement with more recent work on latitudinal angular separation (Dalla et al. 2003).

We chose to concentrate on events that are magnetically well-connected to the observer (flares from the western hemisphere), have low background fluxes, and have moderately strong intensities, which together lead to derived onset times with less than 10 min uncertainity. Delays due to systematic errors are not expected to exceed a few minutes. A total of 18 events met the criteria that the start of the solar release of the protons can be reliably determined from COSTEP data and that the NRH observes the Sun at that time. Out of this set, we study those events (six cases) where two further conditions are satisfied during the time interval when the proton release is inferred to start: (i) a decametric-to-kilometric type III burst is observed; and (ii) a parent active region can be identified by an $\mathrm{H} \alpha$ flare or, if no such event was reported, by a brightening in the $19.5 \mathrm{~nm}$ images taken by SoHO/EIT. These supplementary selection criteria allow us to be reasonably sure that particles accelerated in the corona could escape to interplanetary space and that no essential activity occurred on the far side of the Sun and could therefore be occulted.

\section{Results}

\subsection{Overview: proton measurements}

Parameters of the particle events ( $E_{\max }$ : energy up to which protons are observed, $J_{\max }$ : peak intensities in two adjacent energy ranges, average pitch angle of the earliest arriving protons) are listed in Table 1, together with the speed of the solar wind (measured by Wind/SWE, Ogilvie et al. 1995), the inferred connection longitude of the nominal Parker spiral, and the longitude of the associated flare observed in $\mathrm{H} \alpha$ or EUV. Upper limits of the proton peak intensities are given for events which were not detected in the high energy range or which occurred during the decay of a previous event. More details on the associated flare will be discussed below (see also Table 2). The events are ordered by decreasing peak intensity in the (8-25) MeV range.

The peak intensities can be compared with those in the paper of Cane et al. (1986), where the distinction between "impulsive" and "gradual" SEP events was studied in detail, based on association with short-lasting and long-lasting soft X-ray emission, respectively. Our selection criteria, especially the close association with DH type III bursts, favour impulsive events, which is confirmed by the duration of the associated X-ray burst (see below). The impulsive events of Cane et al. (1986) have peak proton intensities in the (9-23) $\mathrm{MeV}$ band from $10^{-3}$ to $1\left(\mathrm{~cm}^{2} \mathrm{~s} \mathrm{sr} \mathrm{MeV}\right)^{-1}$. The events studied in the present work are comparable to the weaker half of the events in their sample. The events of Cane et al. (2003), studied in an attempt to 

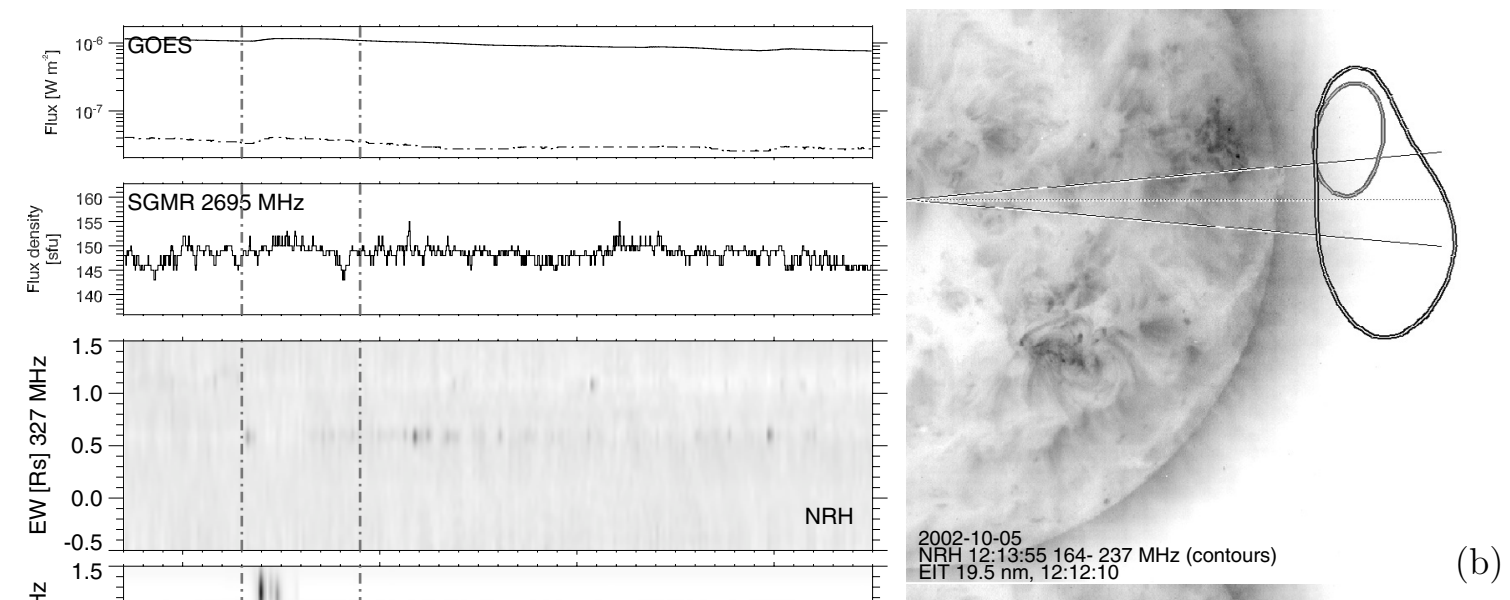

(a)
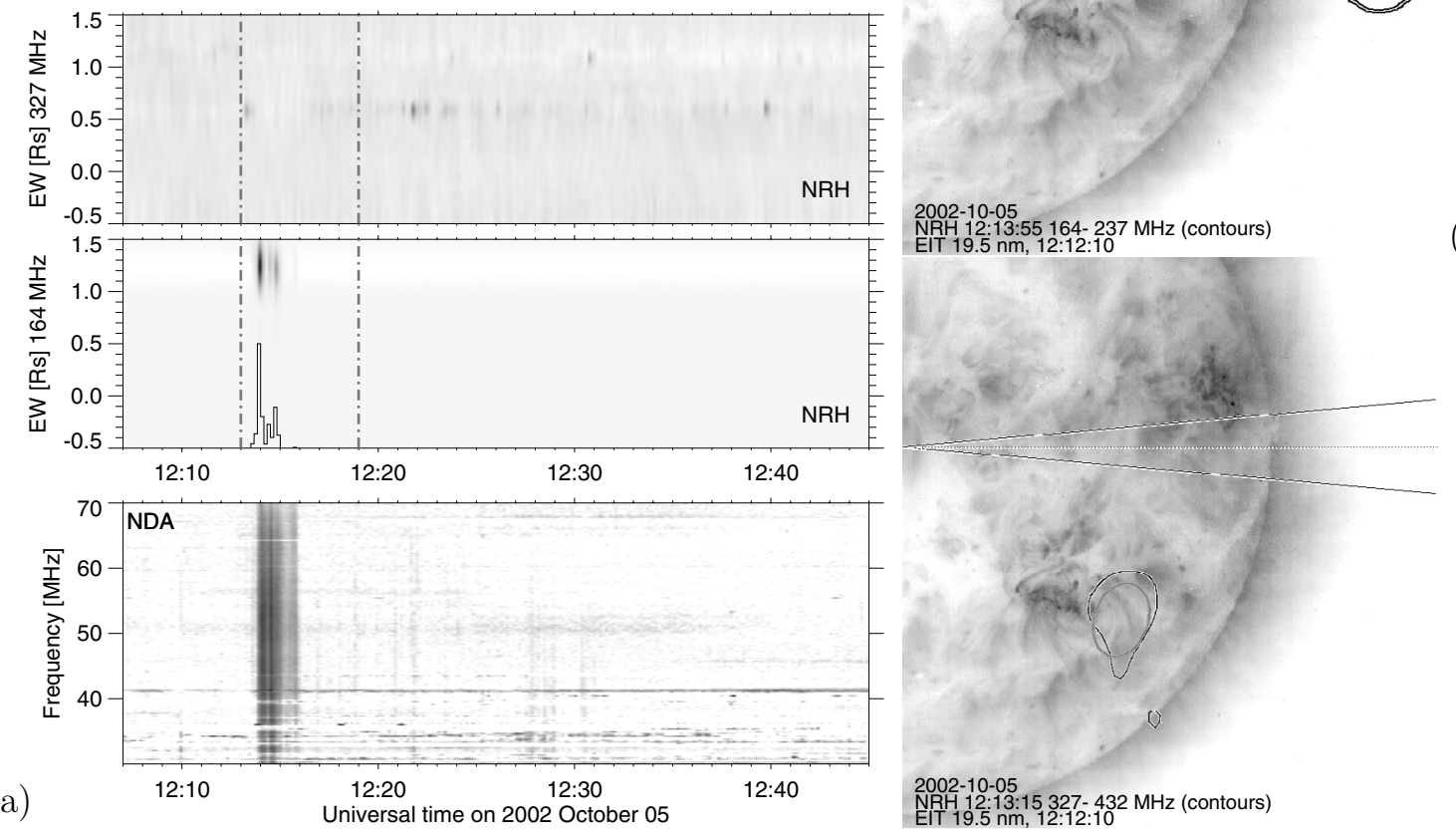

Fig. 2. 2002 October 05: a) time history of (from top to bottom) the whole Sun soft X-ray flux (GOES; two energy bands: 0.1-0.8 nm, upper curve, and 0.05-0.4 $\mathrm{nm}$ ) and centimetric radio flux density (Sagamore Hill), the radio emission at 327 and $164 \mathrm{MHz}$ as a grey-scale plot in the plane time - solar east-west coordinate $(0=$ centre of the disk; Nançay Radioheliograph), the long metric radio spectrum (Nançay Decametric Array). Dark shading means bright emission. b) Contours of the type III source at half maximum intensity at frequencies $237 \mathrm{MHz}$ (inner, smaller contour) and $164 \mathrm{MHz}$ (elongated contour) overplotted on an EIT $19.5 \mathrm{~nm}$ image taken less than 2 min before the radio maps. The EIT image is plotted with a reverse colour table, where dark shading means bright emission, and with a logarithmic scale. The radial lines indicate the central axis and the borders of a CME. Solar north is at the top, west on the right. c) Contours of the sources of weak bursts at 327 and $432 \mathrm{MHz}$ during the release window on top of the same EIT image.

distinguish flare-related particles and CME shock-related ones, are considerably more intense than those in our sample, with peak intensities in the (24-29) $\mathrm{MeV}$ band ranging from 0.08 to $11\left(\mathrm{~cm}^{2} \mathrm{~s} \mathrm{sr} \mathrm{MeV}^{-1}\right.$ (see Cane et al. 2002). We summarise the properties of events in Table 1 as follows:

1. During the events under study protons were accelerated to energies of tens of MeV. In two cases the spectra continued beyond the high-energy limit of the detector.

2. The energy dispersion of proton arrival at the spacecraft was in general ( $5 / 6$ cases) best fit by including a non-zero average pitch angle (see Eq. (1)). The average pitch angles in Col. 5 mean that the travelled distances ranged from the length of the nominal Parker spiral (2002 Oct. 05) to 1.6 times this length (2002 Oct. 20).

3. In four of six events the flare occurred close to the footpoint of the nominal Parker spiral that connects the Sun to the spacecraft. This shows that particles accelerated in the corona have rapid access to the spacecraft. Particular examples will be discussed in the presentation of individual events below (Sect. 3.2 and Appendix).

4. In all of these events, the release time of electrons is in agreement with the assumption of a simultaneous initial release of electrons and protons at the Sun.

\subsection{Individual events}

The relative timing of the initial particle release and signatures of energy release in the corona is illustrated in this section by one event with weak (2002 Oct. 05) and one with strong soft X-ray and microwave signatures. The other events are discussed in the Appendix.

\subsubsection{October 05}

The time histories of broadband radio emission and soft Xrays are plotted in Fig. 2a. The interval within which the proton release is found to start (henceforth referred to as the 
release window) is overplotted as a pair of vertical dashed lines $^{6}$. The figure shows the whole Sun soft X-ray (top panel) and microwave emission (2nd panel from top), the temporal and spatial evolution of the metre wave emission at $327 \mathrm{MHz}$ (92 $\mathrm{cm}$ wavelength, 3rd panel from top) and $164 \mathrm{MHz}$ $(1.83 \mathrm{~m}, 4$ th panel), and the dynamic spectrum at long metreto-decametre waves (bottom panel). The time evolution of the metre wave emission is represented by the $1 \mathrm{D}$ brightness. It is obtained by integrating each map along the solar south-north direction. This way one east-west scan is obtained for each time, in this case using $10 \mathrm{~s}$ integration. The vertical axis gives the east-west coordinate in solar radii, with the zero at the centre of the disk.

The proton release starts during metric-to-decametric emission, which at higher time resolution than used in the figure, appears as a group of type III bursts. Observations by the Tremsdorf spectrograph of the Potsdam Astrophysical Institute $(S G D)$ show the group starting at $270 \mathrm{MHz}$. Its spectral extension to below the low-frequency limit of the NDA suggests that the wave-exciting electron beams escape from the Sun, and that a type III burst would probably have been detected by WAVES at lower frequencies, had data been taken during this time. The NRH observes the type III sources at 164 and $237 \mathrm{MHz}$. The 1D brightness at $164 \mathrm{MHz}$ in Fig. 2a shows the source lies above the western limb (east-west coordinate $>1$ ). The sources at 164 and $237 \mathrm{MHz}$ are overplotted in Fig. $2 \mathrm{~b}$ as iso-intensity contours at half maximum on an EIT image $(19.5 \mathrm{~nm}$; reverse colour scale) taken close to the radio event. The source at $237 \mathrm{MHz}$ has the inner, smaller contour. The $164 \mathrm{MHz}$ contour is elongated due to the presence of two neighbouring sources whose northern component is the low-frequency counterpart of the $237 \mathrm{MHz}$ source. The two sources at $164 \mathrm{MHz}$ suggest we see sections of two different flux tubes that guide electrons outward from an acceleration site in the underlying corona. Their location near the equator confirms the good magnetic connection to the spacecraft inferred from the flare location near the Earth-connected interplanetary field line (Table 1). Since the $237 \mathrm{MHz}$ source coincides in projection with one of the sources at $164 \mathrm{MHz}$, the flux tube is most likely curved towards the observer.

If the type III bursts are emitted at the harmonic of the local plasma frequency, the metric-to decametric type III emission traces electron beams travelling from a region with electron density $2 \times 10^{8} \mathrm{~cm}^{-3}$ at their start $(270 \mathrm{MHz})$ outward to $1 \times 10^{6} \mathrm{~cm}^{-3}$ at the low-frequency end of the NDA. The imaging observations suggest that the electrons are accelerated in this flux tube at some height below the radio source at $237 \mathrm{MHz}$, i.e. at a heliocentric distance below $1.2 R_{\odot}$. At $327 \mathrm{MHz}$ some weak bursts are observed as starting closer to central meridian during the release window (Fig. 2a). It is uncertain if they have any relationship with the type III burst above the limb. The sources at 327 and $432 \mathrm{MHz}$ are overlaid on the same EIT image in Fig. 2c. Although it is uncertain if they reveal electrons

\footnotetext{
${ }^{6}$ We emphasise that the term "release window" refers to an interval of statistical uncertainty around the time when the first escaping protons are released. It does not mean that all particles are released during this interval.
}

that escape from the Sun, the close temporal association with the type III bursts at lower frequencies and the proximity of the southern component of the double source at $164 \mathrm{MHz}$ suggest that a large-scale magnetic field configuration could be involved in this event, including transequatorial loops that interconnect two active regions.

The microwave time profiles show faint fluctuations at $2.7 \mathrm{GHz}$ (Fig. 2a), but no emission above the quiet Sun level at higher frequencies. Microwave bursts at frequencies above a few $\mathrm{GHz}$ are gyrosynchrotron radiation by mildly relativistic electrons of energies between about 50 and several hundreds of $\mathrm{keV}$ in magnetic fields of typically a few hundreds of Gauss. A typical spectrum has its maximum near $10 \mathrm{GHz}$. The flux density depends mainly on the number of energetic electrons and the magnetic field strength in the source. The absence of any remarkable microwave signature in this event implies that none or few mildly relativistic electrons exist in the low corona at the time of the initial proton release. The soft X-ray flux has a time profile typical of impulsive flares, but is also very weak, which indicates a low temperature or low emission measure (i.e. low density) of the heated plasma. The inconspicuous signatures in microwaves and soft X-rays suggest, together with the low starting frequency of the type III bursts, that the energy during this event is released at greater heights than in typical impulsive flares. Notwithstanding, the metric radio emission and proton release occur during the rise of the soft X-ray burst, as is typical of particle acceleration signatures during impulsive solar flares.

No $\mathrm{H} \alpha$ flare was reported in $S G D$. However, the Meudon Heliograph $^{7}$ observed a brightening in the centre and wing of the line between 11:45 and 12:15 UT in the north-western quadrant. The brightening is also shown in the EIT $19.5 \mathrm{~nm}$ image in Figs. $2 b$ and $c$ as a set of dark patches underneath the type III source at $237 \mathrm{MHz}$.

The presumed flux tubes project onto the extent of a narrow, faint, but fast CME observed by LASCO (Table 2). Its central axis and angular span are outlined by the radial lines in Figs. $2 b$ and c. Using the trajectory of the CME front in Yashiro's catalogue, we find that the front of the ejecta is at a projected heliocentric distance of (1.6-2.7) $R_{\odot}$ when the protons and electrons start to be released at the Sun, i.e. above the region where the type III burst emitting electrons were accelerated. Since the proton release window coincides with the radio bursts, we conclude that the first electrons and protons that reach the spacecraft are accelerated at the same time and in the same region behind the front of the ejecta.

\subsubsection{May 01}

The most intense SEP event of our sample occurred on 2000 May 01 (Fig. 3). Like on 2002 Oct. 05, the bright decametric type III burst, which here is observed down to kilometric wavelengths at the Wind spacecraft (two bottom panels of Fig. 3a), occurs during the early rise of the soft X-ray emission, i.e. during the impulsive flare phase. It is associated with a group of metric type III bursts $(164,237 \mathrm{MHz})$ and a more

\footnotetext{
7 cf. daily movies, http: //bass2000.obspm.fr
} 

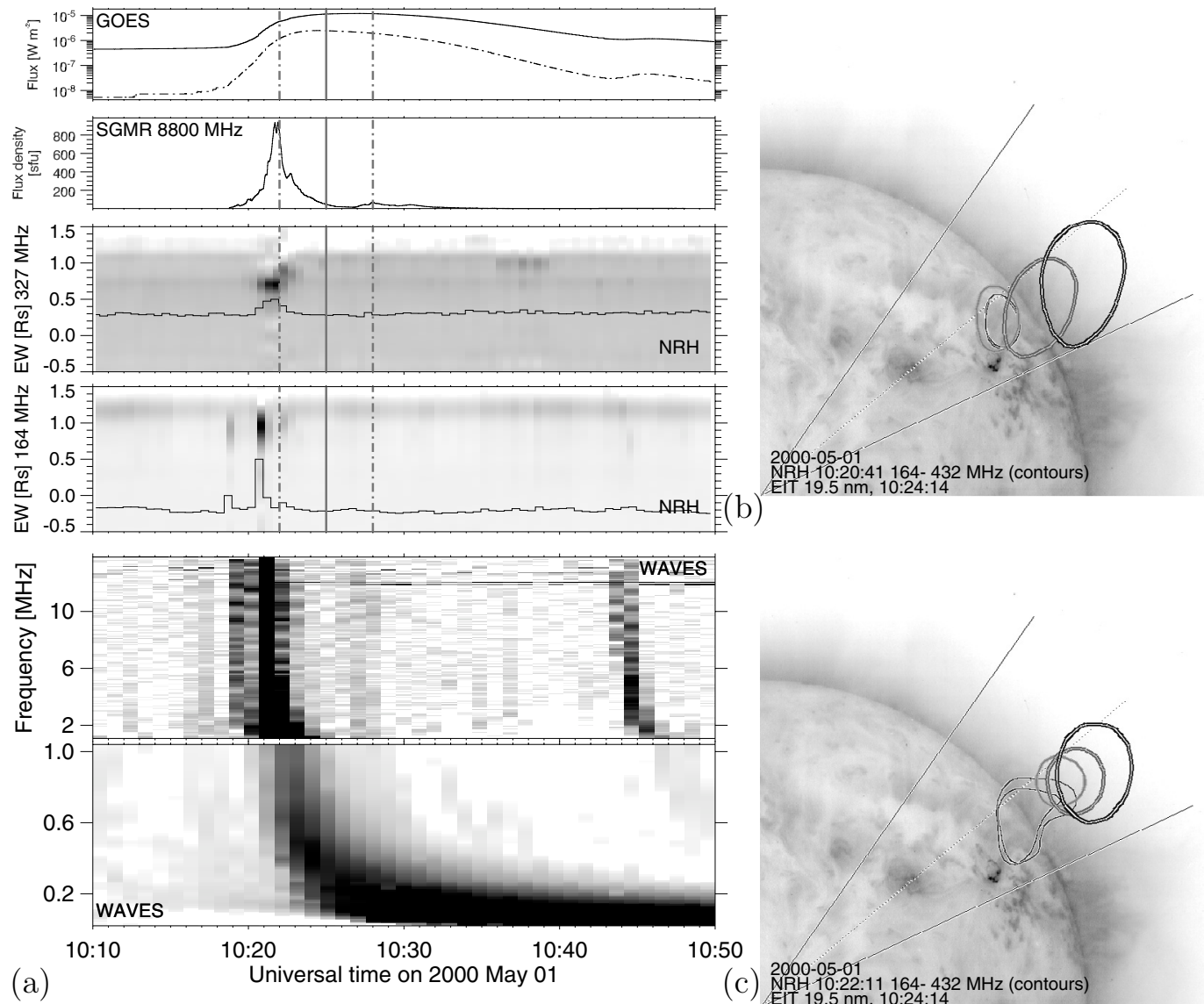

Fig. 3. 2000 May 01: a) time history of (from top to bottom) the whole Sun soft X-ray and microwave emission, the radio emission at 327 and $164 \mathrm{MHz}$ as a grey-scale plot in the plane time - solar east-west coordinate $(0=$ centre of the disk), the decametric-to-hectometric $(\mathrm{DH})$ radio spectrum. Dark shading means bright emission. b) Contours of the radio emission at half maximum intensity at frequencies 432, 410, 327, 237, $164 \mathrm{MHz}$ (order of increasing darkness of the contour and of increasing size) during a burst at 10:20:41 overplotted on a negative EIT 19.5 nm image which shows a brightening (black shading, logarithmic scale) at the base of the radio emitting structure. The radial lines indicate the central axis and the borders of a CME. c) Iso-intensity contours of a burst at 10:22:11 UT superposed on the same EIT image.

smoothly evolving decimetric broadband emission $(327,410$, $432 \mathrm{MHz}$ ) with superposed rapid bursts, which may be metric spikes that are not resolved in the figure due to the $10 \mathrm{~s}$ time integration. The radio emission has a conspicuous microwave component, illustrated by the time history at $8.8 \mathrm{GHz}$ in Fig. 3a. This demonstrates that mildly relativistic electrons are also accelerated in the low corona during this event, unlike 2002 Oct. 05.

The 1D brightness distribution in Fig. 3a shows two successive sources at 327 and $164 \mathrm{MHz}$, the latter being west of the former. The two source configurations are plotted in Figs. 3b and $\mathrm{c}$ on top of an EIT image taken about 2 min. later. The darker the contours, the lower the frequency. The height of the radio sources increases with decreasing frequency, i.e. with decreasing ambient electron density, as expected for a plasma emission process in a nearly radial magnetic flux tube. The two source complexes outline two different structures rooted in the underlying active region where the flare is seen as a compact brightening (i.e. dark spot in the image plotted with reverse colour table) south of the high-frequency radio source. Since the emission consists of continuum and spikes at high frequencies and type III bursts at low frequencies, the structures outlined by the contours in Fig. 3 may be more complex in the low corona than simple open flux tubes. In any case the starting frequency of the type III bursts between 237 and $327 \mathrm{MHz}$ shows that the electrons released into interplanetary space were accelerated in a region where the ambient electron density exceeds $3 \times 10^{8} \mathrm{~cm}^{-3}$, in or above the flaring active region. The radio source locations at 327 and $237 \mathrm{MHz}$ show that this occurs at heights $\lessgtr 0.2 R_{\odot}$ above the photosphere.

The proton release appears to start a few minutes after the onset of the centimetric-to-kilometric radio emission and of the brightest DH type III burst. Such a short delay is not necessarily significant, as it may also be due to systematic errors of the onset time determination. But we note that the release window overlaps with the late metre wave bursts from the western sources. The more detailed plot in Fig. 4 shows the start of the release window at 10:22 UT near the start of the western source at $327 \mathrm{MHz}$. Faint DH type III emission is also observed after the bright burst that dominates in Fig. 3a. The microwave emission starts with the early metric type III bursts and the decimetric broadband emission (cf. $327 \mathrm{MHz}$ ), but overlaps with the late type III bursts west of the former ones (Fig. 4). If the delay of the proton release were significant, it would suggest that in the 2000 May 01 event the first escaping deka-MeV protons are not accelerated with the most energetic electrons in the low 

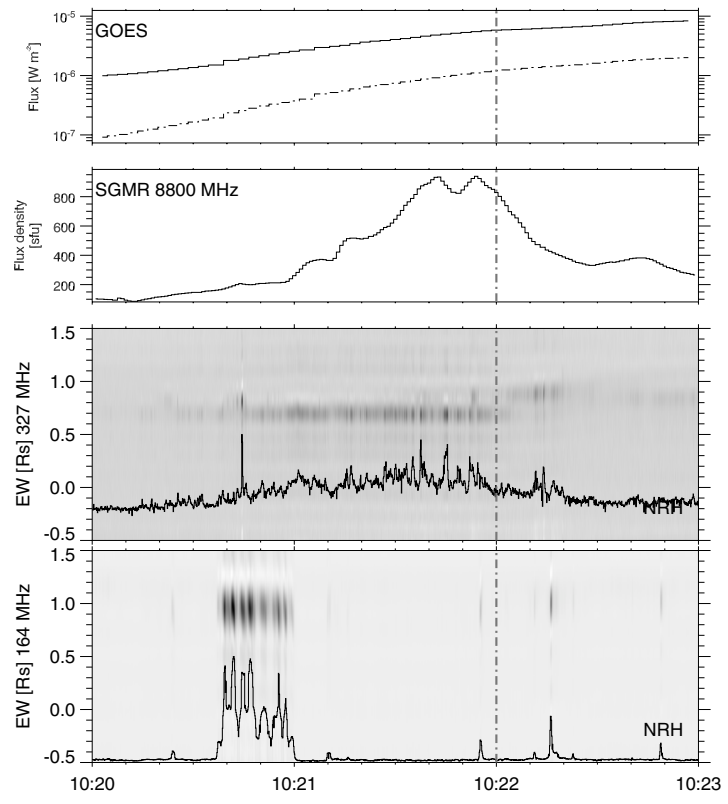

Fig. 4. Soft X-ray, microwave, and metric radio wave emission during the 2000 May 01 event, using $1 \mathrm{~s}$ time resolution of the NRH to clearly distinguish the two source complexes.

corona, but come from later acceleration at slightly different coronal sites. The time scales of electron acceleration, inferred from the duration of individual type III bursts, are on the order of a second.

During this event LASCO observed a narrow CME again whose projected speed exceeded $1000 \mathrm{~km} \mathrm{~s}^{-1}$. Its angular extent is outlined by the radial straight lines in Figs. $3 \mathrm{~b}$ and c. The coronographic observations were discussed by Kahler et al. (2001). The EUV and radio activity occurs within the span or at the southern border of the CME. At the time of the radio emission the CME front is ahead of the $164 \mathrm{MHz}$ radio sources by about $0.5 R_{\odot}$ in projection on the plane of the sky. The onset of the EUV brightening is unclear, but the radio emission most likely occurs after the launch of the CME.

\subsection{Summary: proton release and related activity}

The timing of the particle events and the associated solar activity are listed in Table 2:

1. $\boldsymbol{T}_{\text {release }}$ : the first column gives the inferred release time of protons (He ions for the 2002 Oct. 20 and Dec. 12 events) at the Sun, to which 8 min have been added for comparison with the electromagnetic signatures, and the estimated statistical uncertainty.

2. Flare: the second column lists the importance, position, and start time of the $\mathrm{H} \alpha$ flare or flares that are reported around the proton release time or of the brightening seen at $19.5 \mathrm{~nm}$ by SoHO/EIT. The suffix E after the starting time means that the event was already in progress at the time indicated. The second line of each entry gives the importance, start, and maximum time of the soft X-ray burst observed by GOES in the $(0.1-0.8) \mathrm{nm}$ range, as reported in $S G D$ or read from the data plots.
3. cm-wave burst: Col. 3 gives the peak frequency $\left(v_{\mathrm{m}}\right)$ and the maximum frequency where the centimetric burst was observed $\left(v_{\mathrm{u}}\right.$, within parentheses; RSTN observes up to 15.4 GHz). The second row of each entry contains the peak flux density (in units of $1 \mathrm{sfu}=10^{-22} \mathrm{~W} \mathrm{~m}^{-2} \mathrm{~Hz}^{-1}$ ), start and peak time.

4. m-wave burst: characteristics of the metre wave emissionin in the vicinity of the release window are summarised in Col. 4: spectral type(s), start times $\left(t_{\mathrm{s}}\right)$ and start frequency $\left(v_{\mathrm{s}}\right)$, together with (second line of each entry) a rough indication of the position angle (counterclockwise from solar north) and heliocentric distance (units of the solar radius) of the radio source at $164 \mathrm{MHz}$.

5. DH radio: the type, starting time, and starting frequency of DH radio emission are listed in Col. 5, as observed by WAVES/Wind at frequencies $<14 \mathrm{MHz}$ or - on 2002 Oct. 05, where no WAVES data are available - by the Nançay Decametric Array (NDA). The times refer to the highest frequencies where the bursts could be observed, i.e. in general to $14 \mathrm{MHz}$. When a group of type III bursts is observed, the times of individual bursts or the time interval during which bursts are observed are listed.

6. CME: the last column contains characteristics of the CME: position angle (PA) and width $(W)$ in the first line, speed $(v$; $\left.\mathrm{km} \mathrm{s}^{-1}\right)$, and reference time $\left(t_{0}\right)$ of the CME in the 2nd line. The central position angle and width were measured in the first image showing the CME in the daily movies of the LASCO C2 field of view. Speed and reference time are inferred from a linear fit to the heliocentric distance-time plot of the front of the CME (values from Yashiro's LASCO CME catalogue; evaluation from Fig. $4 \mathrm{~b}$ in Sheeley et al. (2000) for the 1999 Jun. 29 event where the ejecta near the site of the radio emission at $\mathrm{PA}=290^{\circ}$, designated $\mathrm{N} 20 \mathrm{~W}$ in that figure, are part of a halo CME which is more pronounced on the eastern limb). The reference time is the instant when the fitted heliocentric distance of the CME front is equal to the heliocentric distance of the radio source at $164 \mathrm{MHz}$ quoted in Col. 4. The third line of each entry in this column gives the range of heliocentric distances of the front of the CME between the time of the first DH type III burst and the end of the proton release window, computed using the reference time and speed in the previous line. Since only C3 observations are available on 1996 Jul. 09, the reference time and the values of the heliocentric distance of the CME front are uncertain, and are preceded by tildes. In several cases the indicated CME position refers to structures within a more extended CME. The widths in Table 2 are then smaller than in Yashiro's list, where the overall extent of the CME is given.

Type II bursts, which are ascribed to coronal shock waves, do occur in some of these events. Metric type II emission was observed during the proton release window in the 1996 Jul. 09 event (Mann et al. 1999), but at the same time there was emission at higher frequencies that was not caused by the coronal shock (Laitinen et al. 2000). On 2002 Oct. 20, a type II burst was observed well after the release window at frequencies below $100 \mathrm{MHz}$ (Fig. A.4), yet without any 
Table 2. Proton release times ( $T_{\text {release }}$ ) and associated activity: $t_{\mathrm{s}}$ and $v_{\mathrm{s}}$ are starting times and starting frequencies (i.e. high-frequency cutoffs), $t_{\mathrm{m}}$ and $v_{\mathrm{m}}$ the time and frequency of the microwave spectral maximum, $v_{\mathrm{u}}$ the highest microwave frequency where the event is observed (the highest frequency of the RSTN patrol instruments is $15.4 \mathrm{GHz}$ ). The spectral identification at metric and longer wavelengths is "I", "III", "V" for bursts of the respective types, "DCIM" for decimetric continuum emission, possibly comprising spikes, and "N" for noise storm emission.

\begin{tabular}{|c|c|c|c|c|c|}
\hline Protons & Flare & $\mathrm{cm}-\lambda$ radio & $\mathrm{m}-\lambda$ radio & DH radio & CME \\
\hline$T_{\text {release }}$ & $\mathrm{H} \alpha$ imp. pos. $t_{\mathrm{s}}$ & $v_{\mathrm{m}}\left(v_{\mathrm{u}}\right)$ & Type $t_{\mathrm{s}} v_{\mathrm{s}}$ & Type $t_{\mathrm{s}} v_{\mathrm{s}}$ & PA, $W$ \\
\hline (UT) & GOES imp. $t_{\mathrm{s}} t_{\mathrm{m}}$ & flux $t_{\mathrm{s}} t_{\mathrm{m}}$ & $\mathrm{PA}^{\circ} r\left(R_{\odot}\right)$ & & $v, t_{0}$ \\
\hline \multicolumn{6}{|c|}{20000501 (122) } \\
\hline $10: 24$ & EIT N21 W51 10:24E & $8.8(15.4) \mathrm{GHz}$ & III 10:21 $270 \mathrm{MHz}$ & $310^{\circ} 30^{\circ}$ & \\
\hline \multirow[t]{2}{*}{ \pm 3} & M1.1 10:16 10:27 & 950 10:21 10:22 & $305^{\circ} 1.1 R_{\odot}$ & $>14 \mathrm{MHz}$ & $136010: 15$ \\
\hline & & & DCIM 10:21 & $1.6-2.7$ & \\
\hline \multicolumn{6}{|c|}{$19960709(191)$} \\
\hline 09:15 & 1B S11 W30 09:01 & $8.8(15.4) \mathrm{GHz}$ & II 09:11 $400 \mathrm{MHz}$ & III 09:11 > $14 \mathrm{MHz}$ & $273^{\circ} 86^{\circ}$ \\
\hline \multirow[t]{2}{*}{ \pm 5} & X2.6 09:01 09:12 & 2800 09:08 09:09 & IV 09:09 $\leq 800 \mathrm{E}$ & III 09:23 4 MHz & $452 \sim 08: 44$ \\
\hline & & & $260^{\circ} 0.6 R_{\odot}$ & II 09:24@14 MHz & $\sim(1.6-2.0)$ \\
\hline \multicolumn{6}{|c|}{$20021020(293)$} \\
\hline $14: 20$ & SF S13 W63 14:11 & $5.0(15.4) \mathrm{GHz}$ & III 14:11 $230 \mathrm{MHz}$ & III $14: 11>14 \mathrm{MHz}$ & $247^{\circ} 14^{\circ}$ \\
\hline \multirow[t]{4}{*}{ \pm 5} & 1F-1B S15 W18 14:22 & 58 14:11 14:12 & $255^{\circ} 1.0 R_{\odot}$ & & $101114: 00$ \\
\hline & SF S19 W19 14:28 & $\sim 5(8.8) \mathrm{GHz}$ & III+N 14:22 $330 \mathrm{MHz}$ & III $14: 25>14 \mathrm{MHz}$ & $2.0-3.2$ \\
\hline & C5.6 14:08 14:13 & $\sim 3014: 26$ 14:27 & $215^{\circ} 0.4 R_{\odot}$ & & \\
\hline & & & II 14:32@70 MHz & & \\
\hline \multicolumn{6}{|c|}{$19990629(180)$} \\
\hline $12: 51$ & 1F N21 W45 12:40E & $2.7(8.8) \mathrm{GHz}$ & III (?) $12: 40 \geq 327 \mathrm{MHz}$ & III 12:36-12:43 & $292^{\circ} 25^{\circ}$ \\
\hline \multirow[t]{2}{*}{$-10 /+5$} & C2.8 12:40 12:47 & 27 12:42 12:42 & $290^{\circ} 0.9 R_{\odot}$ & $>14 \mathrm{MHz}$ & $(\sim 400$ 12:40) \\
\hline & & & & II 13:00@14 MHz & \\
\hline \multicolumn{6}{|c|}{$20021212(346)$} \\
\hline $12: 41$ & EIT N15 W37 12:48E & no event & III $12: 35460 \mathrm{MHz}$ & III 12:35-12:39 & $290^{\circ} 20^{\circ}$ \\
\hline \multirow[t]{3}{*}{ \pm 3} & C8.3 12:33 12:39 & & $75^{\circ} 1.1 R_{\odot}$ & $>14 \mathrm{MHz}$ & $72312: 20$ \\
\hline & & & III $12: 37-12: 41 \geq 237 \mathrm{MHz}$ & & $1.9-2.4$ \\
\hline & & & $305^{\circ} 0.9 R_{\odot}$ & & \\
\hline \multicolumn{6}{|c|}{$20021005(278)$} \\
\hline $12: 16$ & EIT N09 W63 11:48E & $2.7(2.7) \mathrm{GHz}$ & III 12:13 $270 \mathrm{MHz}$ & (III 12:13-12:16) & $270^{\circ} 10^{\circ}$ \\
\hline \multirow[t]{2}{*}{ \pm 3} & B4. $1 \sim 12: 12$ 12:15 & $<512: 1312: 16$ & $270^{\circ} 1.2 R_{\odot}$ & (NDA 20-70) & 723 12:00 \\
\hline & & & & & $2.1-2.5$ \\
\hline
\end{tabular}

evident relationship to the DH type III bursts closer to the release window. A very faint type II burst was seen by WAVES during the release window on 1999 Jun. 29. It is not contained in the list of type II and IV bursts on the WAVES homepage.

Inspection of Table 2 leads to the following conclusions:

1. Due to the criteria for event selection, the protons are released at the Sun together with the electron beams emitting DH type III bursts within an uncertainty of a few minutes. The type III bursts start between the onset and the maximum of the associated soft X-ray burst. This means that the DH type III bursts of our sample are in general associated with the impulsive flare phase.

2. Most DH type III bursts (5/6) start at frequencies above the high frequency limit of the WAVES receiver at $14 \mathrm{MHz}$, i.e. in regions where the ambient electron density exceeds
$6 \times 10^{5} \mathrm{~cm}^{-3}$, corresponding to a plasma frequency of $7 \mathrm{MHz}$.

3. All DH type III bursts of these groups are accompanied by simultaneous metric radio emission from lower coronal heights, which in 5/6 cases (exception: 1996 Jul. 09) also contains type III emission, i.e. the metric emission is the high-frequency extension of the DH type III bursts. The lowest starting frequency of these is $230 \mathrm{MHz}$; hence, the outward travelling electron beams are accelerated in regions whose ambient electron density is at least $1.6 \times 10^{8} \mathrm{~cm}^{-3}$, corresponding to a plasma frequency of $115 \mathrm{MHz}$.

4. The particle events are flare-associated by selection. The flares range from GOES classes $\mathrm{B}$ to $\mathrm{X}$ and from unobserved to $1 \mathrm{~B}$ in $\mathrm{H} \alpha$. The weaker particle events in the second half of the table, characterised by a low peak intensity 
and a low high-energy cutoff of the proton spectrum, are also weak soft X-ray events.

5. None or only weak microwave emission is detected in three events (1999 Jun. 29, 2002 Oct. 05, and Dec. 12), again the three weakest proton events. This indicates that no or few electrons are accelerated to mildly relativistic energies in the low corona. The weak microwave events are also weak soft X-ray emitters; hence, a relatively small amount of energy is converted in the low corona into heating or particle acceleration during the weak proton events.

6. All these SEP events are accompanied by coronal mass ejections.

- The projected speeds of the CMEs range from 400 to $1400 \mathrm{~km} \mathrm{~s}^{-1}$ with an average near $700 \mathrm{~km} \mathrm{~s}^{-1}$.

- When they first appear within the LASCO C2 field of view, the CMEs have widths ranging from $10^{\circ}$ to $30^{\circ}$, with average $20^{\circ} \pm 8^{\circ}$. Since the wider CME on 1996 Jul. 09 was only observed in the C3 field of view, we cannot decide if it started as a narrow feature or not.

Extrapolation of a straight line fit to the front of the CME implies that the projected heliocentric distance of the CME front is on average $1.7 R_{\odot}$ at the start of the DH type III emission and $2.6 R_{\odot}$ at the end of the proton release window, while the projected heliocentric distance of the metric radio sources does not exceed $1.2 R_{\odot}$.

7. Type II emission revealing extended shock waves in the corona is observed in three events: it is conspicuous at metre wavelengths on $1996 \mathrm{Jul}$. 09, and in a more restricted range on 2002 Oct. 20 (see Appendix, Sect. A.4). Decametric type II emission is observed in two events, but is faint and of small overall spectral width.

\section{Discussion}

We studied the timing of the onset of proton release at the Sun with respect to dynamical processes in the corona in six solar energetic particle events observed by SoHO/COSTEP between 1996 and 2002. The selection criteria, besides common observations with the NRH, were that the solar release time of the protons be accompanied by (i) a decametric-to-kilometric type III burst demonstrating that particles have access to the high corona and interplanetary space; and (ii) an $\mathrm{H} \alpha$ flare or an EUV brightening, which guarantees that no essential activity was missed because it occurred on the far side of the Sun.

\subsection{Proton release time and interplanetary scattering}

The study shows that the initial release times of protons at deka-MeV energies can be inferred from COSTEP with an uncertainty of a few minutes. The best fit to the PHA data is in general not provided by the assumption that the first arriving protons travel strictly along the nominal interplanetary magnetic field. The "average pitch angle" that we introduced as a supplementary degree of freedom into the fitting procedure confirms that even the first arriving protons may be scattered, as suggested by Kallenrode \& Wibberenz (1990) and by Lintunen \& Vainio (2004). In the sample studied here the protons travel paths of lengths ranging from the nominal Parker spiral to 1.6 times this value.

In two of the events the release of electrons between 30 and $500 \mathrm{keV}$ was studied with Wind/3DP observations (Klein et al. 2005). The release windows inferred under the assumption of parallel propagation along the interplanetary magnetic field were 10:14-10:25 UT on 2000 May 01 and 12:35-12:45 UT on 1999 June 29, as compared to 10:21-10:27 UT and 12:41-12:56 UT, respectively, for the deka-MeV protons (see Table 2). The basic difference is that the interplanetary trajectories of the protons, as inferred from the fits to the onset times, were slightly longer than the length of the nominal Parker spiral interplanetary field lines by factors 1.17 and 1.35 , respectively. Krucker \& Lin (2000) found similarly prolonged path lengths in some of their proton events at energies below $6 \mathrm{MeV}$. However, they propose a different interpretation of this result, namely that protons are accelerated by the CME shock and that protons at successively lower energies are released at successively greater heights. Our argument below (Sect. 4.4) that the CME shock does not accelerate the first arriving deka-MeV protons of the present study contradicts this interpretation. We cannot exclude that different processes affect the protons at energies $\leq 6 \mathrm{MeV}$ studied by Krucker \& Lin (2000) and the deka-MeV protons of the present study.

Closer inspection actually shows that the proton release may start a few minutes after the first DH type III burst. Such delayed proton releases of a few minutes can be ascribed to systematic errors of our onset time determination. But the cases of 2000 May 01 and 1996 Jul. 09 suggest that they may also be due to the successive injection of particles in different flux tubes, which are not all connected to the spacecraft. It is well known from type III studies at metre waves (Pick \& Ji 1986) that during one group of bursts electron beams may radiate in different flux tubes within a range of heliocentric angles that may exceed $30^{\circ}$.

\subsection{Sites of particle acceleration in the corona: type III bursts}

By selection, DH type III bursts are found during or near the start of the proton release in all events. In 5/6 events (exception: 1996 Jul. 09) the type III bursts start at metric wavelengths. Given the starting frequencies $\geq 230 \mathrm{MHz}$, the escaping electron beams are accelerated in regions with typical plasma densities above $10^{8} \mathrm{~cm}^{-3}$, a limit corresponding to harmonic plasma emission. Of course, the acceleration region may be remote from the site where the outward propagating electron beam develops a positive slope in the distribution function and becomes able to generate Langmuir waves. However, the relevant distance over which this occurs was estimated to be small for short electron releases typical of type III bursts $(\leq 25000 \mathrm{~km})$ by Raoult et al. (1990). Aschwanden \& Benz (1997) estimated that the relevant length is still smaller, perhaps as low as $1000 \mathrm{~km}$ (also see Aschwanden 2002). Although this value is model-dependent, it is likely that the type III emitting electrons are accelerated close to the region where they start to emit type III bursts, i.e. at heights above $0.1 R_{\odot}$ and 
below $0.5 R_{\odot}$ above the photosphere. The proton release on 1996 Jul. 09 is accompanied both by a type II burst and by the onset of broadband decimetric-to-metric continuum emission, pointing to an origin at similar coronal heights (cf. also Laitinen et al. 2000).

If the above estimates of the height of the acceleration region and the ambient electron density are correct, the escaping electron beams - and by inference the escaping deka-MeV protons - are accelerated at greater heights than the electrons emitting the hard X-ray and microwave emission during flares. Using either time-of-flight analyses at radio and hard $\mathrm{X}$-ray wavelengths or the identification of upward and downward travelling electron beams in radio spectra, Aschwanden and coworkers (see Aschwanden 2002, and references therein) conclude that acceleration regions of energetic electrons during flares typically have ambient densities $\left(10^{9}-10^{10}\right) \mathrm{cm}^{-3}$, although a minority of observations is also consistent with greater heights and lower densities (see also Klein et al. 1997). The consistently low starting frequency of the type III bursts in our sample suggests that the escaping electrons come from the upper parts of extended acceleration regions in the corona, in agreement with a scenario envisaged, for instance, by Lin (1985, 1993) and Cliver \& Kahler (1991).

\subsection{Acceleration of deka-MeV protons and flare-related electron acceleration}

The SEP events of this study are associated with flares from a broad range of categories from GOES classes B to X. They thus involve different amounts of energy deposition in the low corona, ranging from weak to conspicuous. There is a trend that the most energetic flares produce the most energetic proton events, since the two flares of classes $M$ and $X$ and the three events associated with non-thermal microwave emission are those with the highest energies of escaping protons and with the highest peak intensities. But three of the six SEP events are not accompanied by microwave gyrosynchrotron emission that would reveal mildly relativistic electrons in the low corona between one and a few times $10^{4} \mathrm{~km}$ above the photosphere, although protons up to $20 \mathrm{MeV}$ are observed by COSTEP. Clearly, SEP events can be associated with unconspicuous flares and with flares that show virtually no signature of particle acceleration in the low corona; yet, the type III emission demonstrates that electron beams escape from the corona irrespective of the microwave counterpart. The presence of type III emission at metre and longer waves with weak or no signatures of electron acceleration in the low corona is well known (Aschwanden et al. 1985; Lin 1985). This can be expected, since type III bursts are due to electrons of lower energies $(\lessgtr 10 \mathrm{keV})$ than microwave gyrosynchrotron emission ( $>50 \mathrm{keV})$. Furthermore, type III emission is a coherent process whose intensity does not linearly depend on the electron number. This makes it readily understandable that type III bursts are much more frequent than microwave bursts. But well-defined SEP events at deka-MeV energies are not frequent, and certainly not a common counterpart of wellconnected type III burst sources. Kahler et al. (2001) therefore suggested that such SEP events accompany particularly energetic flares, which, however, is contradicted by our observations. They argue in favour of distinct, although somehow related, processes of energy conversion which produce, on the one hand, energetic particles interacting in the low corona and on the other hand, protons and electron beams which escape into interplanetary space. As a consequence, the SEP events discussed here may be termed "flare associated", but this term must not be understood to mean protons produced in the same acceleration region as the interacting particles. They may be particularly energetic "high coronal flares" in the terminology of Cliver \& Kahler (1991).

This conclusion on a relatively high and tenuous acceleration region of escaping energetic particles has some bearing on the interpretation of charge states. The energy-dependent charge states of heavy ions discovered with the ACE spacecraft (Möbius et al. 2003) can be understood in terms of collisional stripping (Kocharov et al. 2001). However, the observations imply that the product of ambient density and residence time in the acceleration region be at least $10^{10} \mathrm{~cm}^{-3} \mathrm{~s}$ (Klecker et al. 2005). If the short acceleration times of the type III emitting electron beams $(\sim 1 \mathrm{~s})$ also characterise the ion acceleration, the densities of the acceleration region inferred from the present study seem too low by at least one order of magnitude, while the higher densities in the acceleration regions of the hard $\mathrm{X}$-ray emitting electrons are consistent. We do not know if the events of our sample also display the energy dependent charge states for heavy ions analysed by Klecker and coworkers, but there may be a conflict of interpretations.

\subsection{CME association}

The six SEP events are accompanied by coronal mass ejections with speeds ranging from 400 to $1300 \mathrm{~km} \mathrm{~s}^{-1}$. The five CMEs observed by C2/LASCO above a heliocentric distance of $2 R_{\odot}$ are narrow (average: $20^{\circ}$ ) when they first appear in the coronagraph field of view, and comprise the flaring active region and the radio sources. The speeds of the CMEs suggest that most drive a shock. Type II radio bursts in three events demonstrate indeed that shocks exist, although it is not proven that they are driven by the CMEs. It is remarkable that no type II burst is observed with the fastest CME (2000 May 01), while metric and decametric type II emissions accompany a slow one (1996 Jul. 09).

The shocks do not seem to play a part in the acceleration of the initial protons dicussed here, since the timing with respect to the rise of the CME shows that the first particles are accelerated behind the front of the CME, not at the CME's bow shock. This means that the shocks (i) do not intercept the Earth-connected interplanetary magnetic field line, (ii) do not interact with the deka-MeV protons observed by COSTEP, or (iii) are not yet formed at the time when the first particles escape to the interplanetary space. (i) might be suggested since at a mean width of $20^{\circ}$, the CMEs associated with the present proton events are much narrower than average CMEs. Such CMEs were already discussed by Kahler et al. (2001) in association with "impulsive" particle events. But since the 
CMEs of our sample accompany flares near the nominal connection longitude, the narrowness should not be an obstacle in the early phase of the particle event. Possibility (iii), suggesting that the shock develops at greater altitude, is supported by the model of Mann et al. (2003) that predicts a maximum of the Alfvén speed around (3-4) $R_{\odot}$ above the photosphere (cf. also Gopalswamy et al. 2001) and most efficient shock acceleration beyond that point. Table 2 indeed shows that the CME fronts in our sample are at a similar projected height at the end of the proton release window. In this case one would expect that shock acceleration contributes to later phases of at least some particle events in our sample. Such a scenario was proposed by Cliver (1996), Kocharov \& Torsti (2002) and Cane et al. (2003). However, there is no agreement on the maximum of the magnetosonic speed at the coronal heights of interest here; e.g., the model of Wang (2000), where the fast magnetosonic speed is computed by a current-free extrapolation of photospheric field measurements, predicts a rapid decrease with height. If this prediction is correct, the most probable interpretation of our observations is that the first escaping protons do not interact with the CME shock.

Kahler et al. (2001) proposed a special acceleration scenario for impulsive SEP events associated with narrow CMEs, where both the accelerated ions and the CME material escape along open magnetic flux tubes. The narrow CME is considered as the reconnection jet, as opposed to the closed magnetic field configuration of broad CMEs. However, Gilbert et al. (2001) and Dobrzycka et al. (2003) did not find convincing evidence that narrow CMEs were fundamentally different from the broader ones, and suggested that the narrow CMEs were initially closed magnetic flux, too. The present study contradicts the scenario of Kahler et al. (2001) through two observations. First, the plane-of-the sky trajectory of the CMEs in our sample, provided by Yashiro's CME catalogue, shows no indication of a ballistic trajectory up to heliocentric distances of $\sim 20 R_{\odot}$, as would be expected if the ejected material were accelerated impulsively in the low corona. Second, the timing of the initial proton release shows that their acceleration must lag behind the launch of the mass ejection, whose front is well above the inferred acceleration region. Nevertheless, the type III spectra show no signature of disturbance by the CME, which suggests that the radio emitting electrons and, by inference, the deka-MeV protons escape along open magnetic flux tubes, which existed already before the launch of the CME.

\section{Summary and conclusion}

In summary, the early proton releases during the solar energetic particle events in this study

- accompany flares and CMEs;

- accompany electron beams escaping into the interplanetary space;

- are associated with different kinds of flares whose radiative signatures in the low corona range from weak to strong;

- probably result from particle acceleration at greater heights than those where the interacting particles are usually produced, but behind the front of the CME.
A plausible site of acceleration is the magnetically stressed corona in the aftermath of the mass ejection, e.g. where the perturbed magnetic field is relaxed through magnetic reconnection. Remaining uncertainities in solar energetic particle onset timing can be further reduced with appropriate energetic particle spectrometers on near-Sun spacecraft, such as the planned ESA Solar Orbiter mission.

Acknowledgements. The SOHO/COSTEP project is supported under grant No. 50 OC 0105 by the German Bundesminister für Bildung und Forschung (BMBF) through the Deutsches Zentrum für Luftund Raumfahrt (DLR). SoHO is a project of international cooperation between ESA and NASA. The Nançay Radio Observatory is funded by the French Ministry of Education, the CNRS, and the Région Centre. This work benefited from the data of EIT and LASCO aboard SoHO, SXT aboard Yohkoh (data provided by SURF), the radio experiment WAVES aboard Wind, the radio spectrographs in Moscow (IZMIRAN), Potsdam-Tremsdorf, Nançay, Ondřejov and Zurich through the respective web-pages, and from the RSTN network through the NOAA webpage (courtesy H. Coffey). H. Aurass (Potsdam) and A. Lecacheux (Meudon) provided detailed data from the Tremsdorf (OSRA) and Nançay (NDA) radio spectrographs, A. Magun (Bern) from the University of Bern microwave patrol instruments, C. Marqué (Washington) from LASCO/SoHO. Extensive use was made of the LASCO/SoHO catalogue of CMEs generated and maintained by the Center for Solar Physics and Space Weather, the Catholic University of America, in cooperation with the Naval Research Laboratory and NASA. KLK acknowledges S. Hoang (Meudon) for discussions on the WAVES data, and P. Zarka (Meudon) for help with NDA software. We acknowledge helpful comments by the referee and by the language editor.

\section{References}

Aschwanden, M. J. 2002, Space Sci. Rev., 101, 1

Aschwanden, M. J., \& Benz, A. O. 1997, ApJ, 480, 825

Aschwanden, M. J., Wiehl, H. J., Benz, A. O., \& Kane, S. R. 1985, Sol. Phys., 97, 159

Bougeret, J.-L., Kaiser, M. L., Kellogg, P. J., et al. 1995, Space Sci. Rev., 71, 231

Brueckner, G. E., Howard, R. A., Koomen, M. J., et al. 1995, Sol. Phys., 162, 357

Cane, H. V., McGuire, R. E., \& von Rosenvinge, T. T. 1986, ApJ, 301, 448

Cane, H. V., Erickson, W. C., \& Prestage, N. P. 2002, J. Geophys. Res., 107,1315

Cane, H. V., von Rosenvinge, T. T., Cohen, C. M. S., \& Mewaldt, R. A. 2003, Geophys. Res. Lett., 30, 5

Cliver, E., \& Kahler, S. 1991, ApJ, 366, L91

Cliver, E. W. 1996, in High energy solar physics, ed. R. Ramaty, N. Mandzhavidze, \& X.-M. Hua, American Institute of Physics Conf. Ser., 45

Dal Lago, A., Schwenn, R., Stenborg, G., \& Gonzalez, W. D. 2003, Adv. Space Res., 32, 2619

Dalla, S., Balogh, A., Krucker, S., et al. 2003, Ann. Geophys., 21, 1367

Delaboudinière, J. P., Artzner, G. E., Brunaud, J., et al. 1995, Sol. Phys., 162, 291

Dobrzycka, D., Raymond, J. C., Biesecker, D. A., Li, J., \& Ciaravella, A. 2003, ApJ, 588,586

Dryer, M., Andrews, M. D., Aurass, H., et al. 1998, Sol. Phys., 181, 159 
Garcia, H. A. 1994, Sol. Phys., 154, 275

Gilbert, H. R., Serex, E. C., Holzer, T. E., MacQueen, R. M., \& McIntosh, P. S. 2001, ApJ, 550, 1093

Gopalswamy, N., Lara, A., Kaiser, M. L., \& Bougeret, J.-L. 2001, J. Geophys. Res., 106, 25261

Hucke, S., Kallenrode, M. B., \& Wibberenz, G. 1992, Sol. Phys., 142, 143

Kahler, S., Reames, D., \& Sheeley, N. 2001, ApJ, 562, 558

Kahler, S. W. 1996, in High energy solar physics, ed. R. Ramaty, N. Mandzhavidze, \& X.-M. Hua, American Institute of Physics Conf. Proc., 374, 61

Kallenrode, M. 1993, J. Geophys. Res., 98, 5573

Kallenrode, M. B., \& Wibberenz, G. 1990, in Proc. 21st ICRC, 5, 229

Kallenrode, M. B., \& Wibberenz, G. 1991, ApJ, 376, 787

Kerdraon, A., \& Delouis, J. 1997, in Lecture Notes in Physics, Coronal Physics from Radio and Space Observations, ed. G. Trottet (Springer), 483, 192

Klassen, A., Karlický, M., Aurass, H., \& Jiřička, K. 1999, Sol. Phys., 188,141

Klecker, B., Moebius, E., Popecki, M. A., et al. 2005, Adv. Space Res., submitted

Klein, K.-L., Aurass, H., Soru-Escaut, I., \& Kalman, B. 1997, A\&A, 320,612

Klein, K.-L., Krucker, S., Trottet, G., \& Hoang, S. 2005, A\&A, 431, 1047

Klein, K.-L., \& Trottet, G. 2001, Space Sci. Rev., 95, 215

Kocharov, L., \& Torsti, J. 2002, Sol. Phys., 207, 149

Kocharov, L. G., Kovaltsov, G. A., \& Torsti, J. 2001, ApJ, 556, 919

Krucker, S., \& Lin, R. P. 2000, ApJ, 542, L61

Laitinen, T., Klein, K.-L., Kocharov, L., et al. 2000, A\&A, 360, 729

Lecacheux, A. 2000, in AGU Monograph, Radio Astronomy at Long Wavelengths, ed. R. Stone, K. Weiler, M. Goldstein, \& J.-L. Bougeret, 119, 321

Lin, R. P. 1985, Sol. Phys., 100, 537

Lin, R. P. 1993, Adv. Space Res., 13, 265
Lintunen, J., \& Vainio, R. 2004, A\&A, 420, 343

Möbius, E., Popecki, M., Klecker, B., et al. 2002, Adv. Space Res., 29, 1501

Möbius, E., Cao, Y., Popecki, M. A., et al. 2003, in Proc. 28th ICRC, 3273

Mann, G., Aurass, H., Voigt, W., \& Paschke, J. 1992, in Coronal Streamers, Coronal Loops, and Coronal and Solar Wind Composition, ESA SP No. 348, 129

Mann, G., Classen, H. T., Motschmann, U., Kunow, H., \& Dröge, W. 1999, Ap\&SS, 264, 489

Mann, G., Klassen, A., Aurass, H., \& Classen, H.-T. 2003, A\&A, 400, 329

McDonald, F. B., \& Ludwig, G. H. 1964, Phys. Rev. Lett., 13, 783

Müller-Mellin, R., Kunow, H., Fleissner, V., et al. 1995, Sol. Phys., 162,483

Neugebauer, M., Liewer, P. C., Smith, E. J., Skoug, R. M., \& Zurbuchen, T. H. 2002, J. Geophys. Res., 107, SSH 13

Neustock, H. H., Wibberenz, G., \& Iwers, B. 1985, in Proc. 19th ICRC, 4, 102

Ogilvie, K. W., Chornay, D. J., Fritzenreiter, R. J., et al. 1995, Space Sci. Rev., 71, 55

Pick, M., \& Ji, S. 1986, Sol. Phys., 107, 159

Pick, M., Maia, D., Kerdraon, A., et al. 1998, Sol. Phys., 181, 455

Posner, A., \& Hassler, D. M. 2004, in EOS Trans. AGU, Fall Meeting Suppl., Abstract SH51A-0262

Raoult, A., Mangeney, A., \& Vlahos, L. 1990, A\&A, 233, 229

Reames, D. V. 1999, Space Sci. Rev., 90, 413

Reames, D. V. 2002, ApJ, 571, L63

Sheeley, N. R., Hakala, W. N., \& Wang, Y. M. 2000, J. Geophys. Res., 105, 5081

Tsuneta, S., Acton, L., Bruner, M., et al. 1991, Sol. Phys., 136, 37

Tylka, A. J., Cohen, C. M. S., Dietrich, W. F., et al. 2003, in Proc. 28th ICRC, 3305

Wang, Y.-M. 2000, ApJ, 543, L89 


\section{Online Material}


K.-L. Klein and A. Posner: Release of deka-MeV protons and associated activity, Online Material p 2

\section{Appendix A: Other events}

\section{A.1. 1999 June 29}

On 1999 Jun. 29, three potential sources of electron beams were identified in the metre wave images (Fig. A.1a): two noise storms, one near central meridian, the other near the western limb, which existed well before and after the proton release window, and a group of type III bursts seen near the western limb during the release window. The NDA (20-70 MHz) observes a storm of relatively weak type III emission during several hours around the release window, and some bursts are also identified in the WAVES spectrum. A much brighter group of DH type III bursts occurs around 12:40 UT. The brightest are the low-frequency counterparts of distinct metric type III emission during the proton release window at a site slightly west of the western noise storm. Figure A.1b shows the metric radio sources of the eastern noise storm (near the central meridian) and of the type III bursts, the latter near the western limb within the cone delimiting the associated narrow CME that occurs in the aftermath of a large halo CME discussed by Sheeley et al. (2000) and Dal Lago et al. (2003).

When the low-frequency edge of the type III burst arrives at the Wind spacecraft, Langmuir waves are observed (not shown in the figure), which demonstrate that the electron beams emitting the type III burst reach the Earth and that the spacecraft intercepts them. The type III emitting electrons and the deka-MeV protons seen by COSTEP thus travel within the same flux tube. Since the type III emission extends to $327 \mathrm{MHz}$, the ambient density in the acceleration region exceeds $3 \times 10^{8} \mathrm{~cm}^{-3}$ (plasma frequency $164 \mathrm{MHz}$ ). A short, weak microwave burst with very low peak frequency (Table 2) accompanies the type III emission, pointing to a small amount of electrons being accelerated in the low corona. A narrow CME is associated with this event. Its borders and central axis are indicated by the radial straight lines in Fig. A.1b.

\section{A.2. 2002 December 12}

On 2002 Dec. 12, metric type III bursts start a few minutes before the proton release window (Fig. A.2a). As seen in the 1D brightness at 327 and $164 \mathrm{MHz}$, the earliest bursts come from the eastern hemisphere. The type III emission in the western hemisphere begins a minute before the proton release window, but the brightest burst is within the release window. The dark patches in the bottom panel of Fig. A.2b (13:40 UT-14:50 UT) show Langmuir waves starting with the arrival of the low-frequency edge of the type III burst at Wind. The highest frequency of the entire group of type III bursts is reported as $460 \mathrm{MHz}$ (Tremsdorf Obs.). But since the western bursts are not seen by the NRH at $327 \mathrm{MHz}$, their starting frequency lies between 327 and $237 \mathrm{MHz}$. The metric type III sources are overplotted on an EIT $19.5 \mathrm{~nm}$ image in Fig. A.2c. An EUV brightening occurs in the trailing part of the parent active region (NOAA 10213), far from the well-connected nominal interplanetary field line. The leading part, connected to the EUV brightening by large loops seen in the EIT image, is at $\mathrm{W} 52^{\circ}$, at $11^{\circ}$ from the nominal Earth-connected field line.
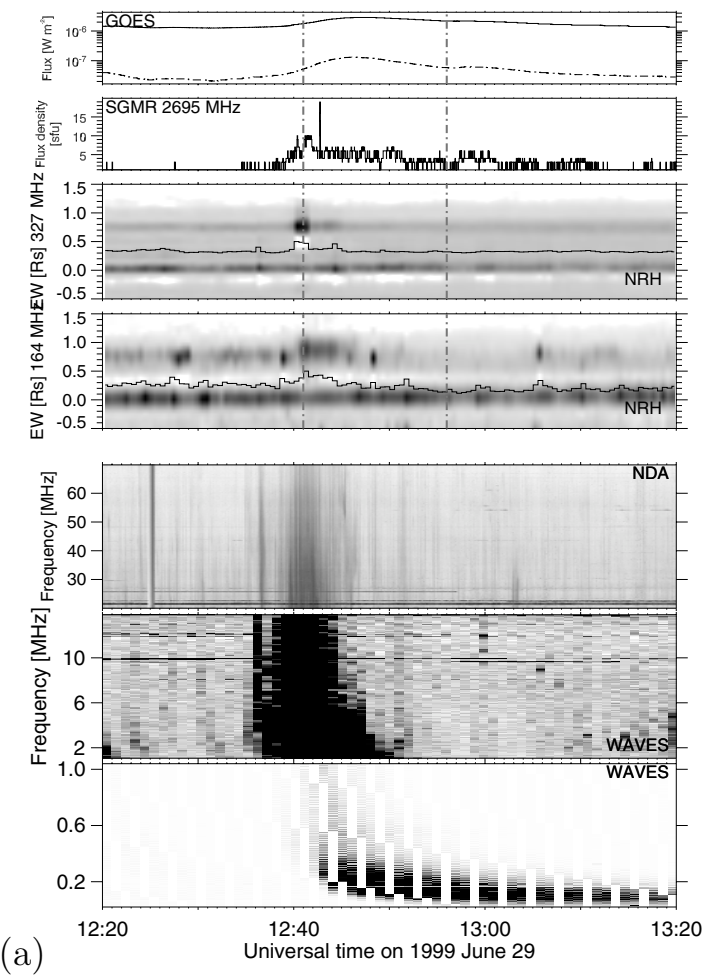

a)

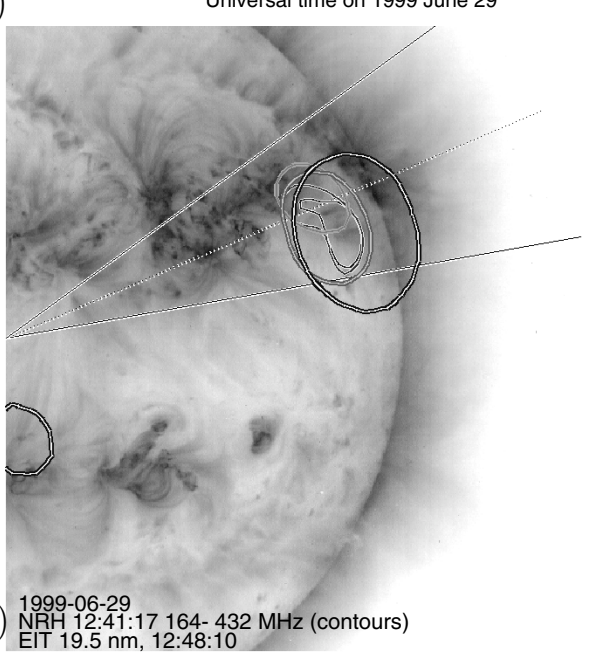

Fig. A.1. 1999 June 29: a) time history of soft X-ray and radio emission; b) iso-intensity contours at half maximum of the sources of type III (north-western source) and noise storm emission (southeastern source). See Fig. 3 for more details. The white rectangles in the bottom panel of a) are instrumental artefacts.

The metric radio sources seem to overlie these loops, but the accuracy of position measurement is weak in December, due to refraction in the Earth's ionosphere. The unambiguous coincidence of the DH type III emission with Langmuir waves on its low-frequency side and metric type III bursts from the vicinity of these loops on the high frequency side clearly shows that the Earth is connected with the acceleration region of the electron beams, which thus propagate in the same flux tube as the protons. While RSTN (Fig. A.2a) registered both a gradual rise of the microwave emission that started well before the proton release window and a slight hump in the flux density curve around the soft X-ray maximum, no emission from mildly 
K.-L. Klein and A. Posner: Release of deka-MeV protons and associated activity, Online Material p 3
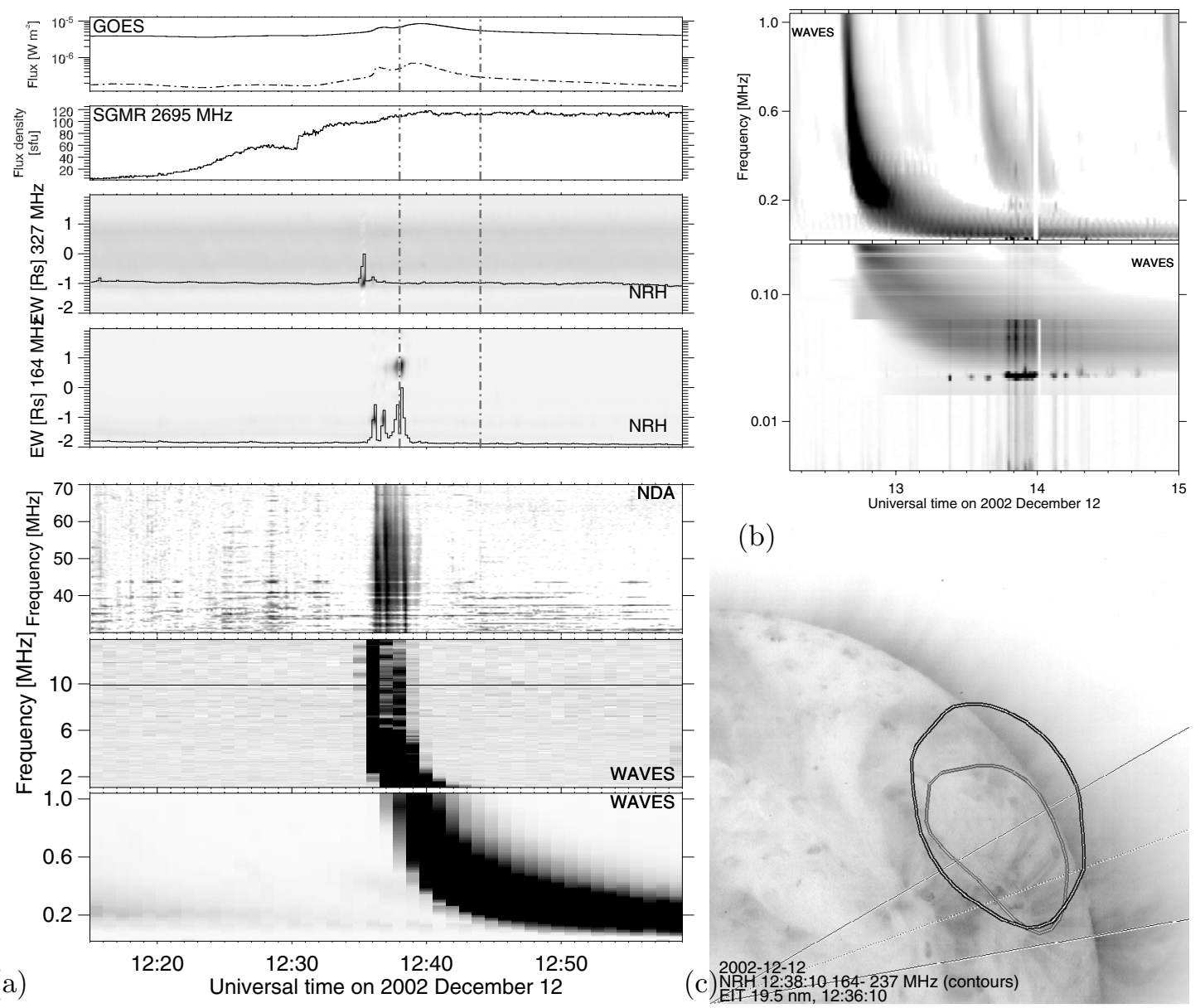

(b)

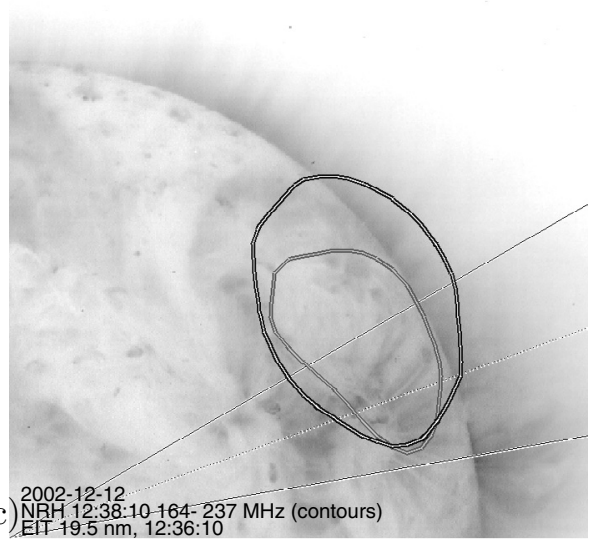

Fig. A.2. 2002 December 12: a) time history of soft X-ray and radio emission; b) Wind/WAVES dynamic spectrum of the type III burst and associated Langmuir waves (dark patches near $20 \mathrm{kHz}$ ); c) iso-intensity contours at half maximum of the source of type III emission at 237 (inner contour) and 164 MHz. See Fig. 3 for more details.

relativistic electrons is observed with the proton release. A narrow $\mathrm{CME}$ is observed at the position angle of the flare and the metric radio sources, within the pair of radial lines in Fig. A.2c.

\section{A.3. 1996 July 09}

The particle release is associated with the most intense soft $\mathrm{X}$-ray burst of our sample, with bursts at gamma-ray, hard $\mathrm{X}$-ray and microwave frequencies, and with various types of metric radio emission. The hard X-ray and microwave emissions show efficient acceleration of electrons in the low corona up to mildly relativistic energies, while the gamma-rays reveal deka-MeV protons interacting with the chromosphere or low corona (Laitinen et al. 2000). These emissions occur slightly before and at the early onset of the proton release into the interplanetary space as inferred from COSTEP (Table 2) and independently from the ERNE instrument on SoHO (Laitinen et al. 2000). The latter authors performed a detailed analysis of the release of electrons and protons during this event, using transport models. This is beyond the scope of our present study of the initial particle release to space.

The proton release window includes the peak of the soft $\mathrm{X}$-ray burst, the peak and decay of the microwave burst, a type II burst (the bright metric radio emission in Fig. A.3a; Dryer et al. 1998; Mann et al. 1999; Klassen et al. 1999) and the onset of long lasting dm-to-m wave type IV emission (the grey band near the western limb in Fig. A.3a). Characteristic configurations of the type II and type IV bursts are shown in the snapshots of Figs. A.3b and A.3c, respectively, on top of a Yohkoh-SXT image taken 11 min earlier. This event does not allow us to unambiguously identify the coronal counterpart of the proton release, because both a type II burst signalling shock acceleration and type IV continuum emission that reveals timeextended electron acceleration over an extended height range at lower altitudes occur during the release window. Laitinen et al. (2000) identified electron releases starting at 09:17 UT, which is within the proton release window in Table 2, and pointed to the close connection between the initial release of electrons and protons with broadband radio emission as a tracer of acceleration processes at lower heights than the shock outlined by the type II burst. Time-extended subsequent proton acceleration was attributed to processes at greater coronal heights, where CME-induced perturbations in the high corona (not necessarily shock waves) were argued to become important.

Since only LASCO/C3 observations (heliocentric distances $\geqslant 6 R_{\odot}$ ) are available (Dryer et al. 1998; Pick et al. 1998), the 

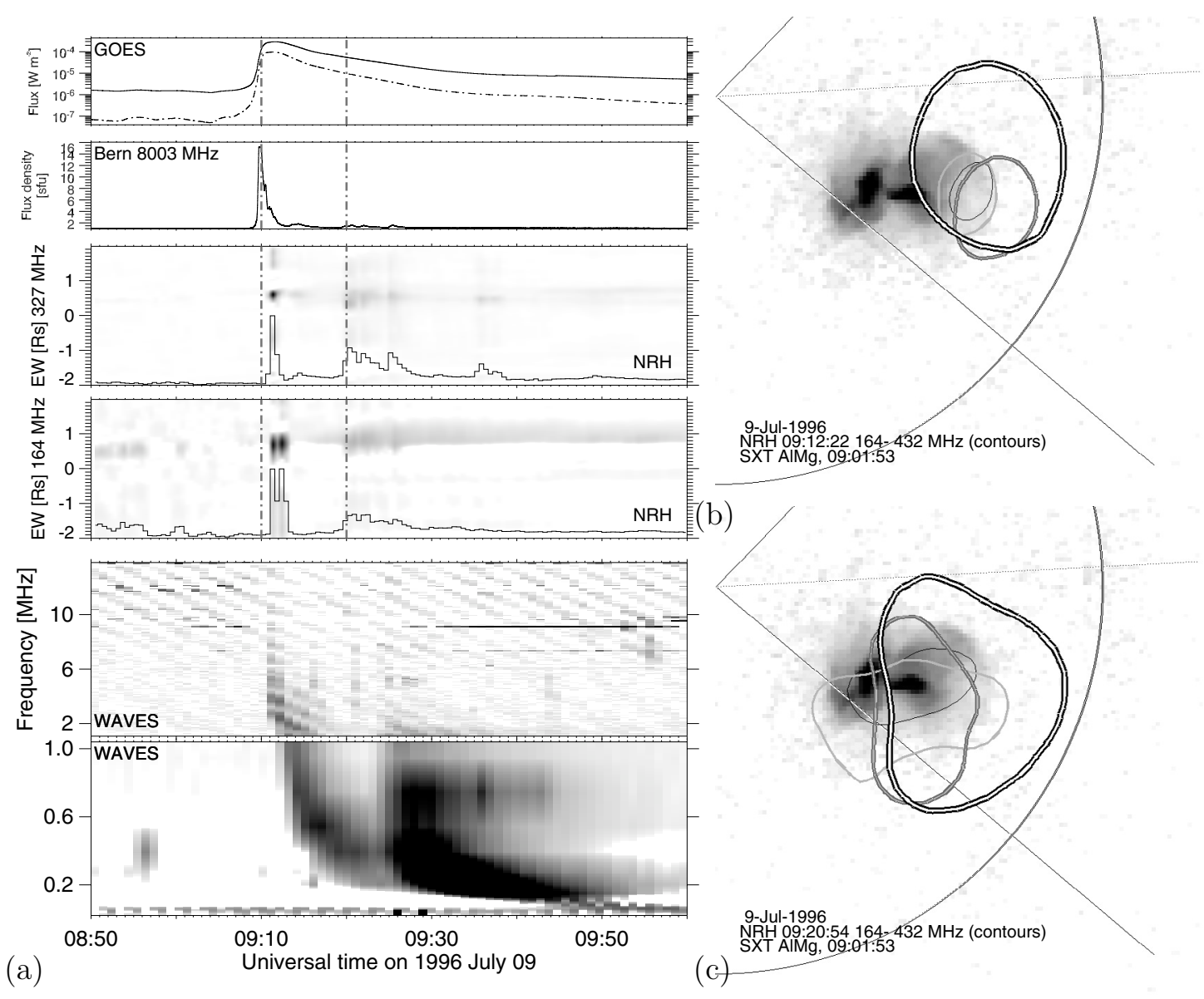

Fig. A.3. 1996 July 9: a) time history of soft X-ray and radio emission; in the WAVES spectrum horizontal lines and inclined periodic features at frequencies above $9 \mathrm{MHz}$ are not of solar origin. The first type III burst is associated with a metric type II burst that stands out in the $\mathrm{NRH}$ plots; b) overlay of radio contours during a bright type II burst $(432,327,237,164 \mathrm{MHz})$ on a Yohkoh/SXT AlMg filter image early during the associated flare (no EIT images available); c) idem during the type IV continuum. See Fig. 3 for more details.

comparison of radio source positions and the CME front is much more ambiguous than in the other events of our sample. The values in Table 2 are consistent with the initial acceleration of escaping particles behind the CME front.

\section{A.4. 2002 October 20}

The first protons are released close to two DH type III bursts, one of them occurring a few minutes before the release window, the other at its end (Fig. A.4a). The first type III burst extends into metric wavelengths, but is not seen at higher frequencies than $164 \mathrm{MHz}$ by the NRH. This agrees with the starting frequency of $230 \mathrm{MHz}$ reported by Tremsdorf Observatory $(S G D)$. Sagamore Hill observes a distinct, albeit weak, impulsive microwave burst at frequencies between 1.4 and $15.4 \mathrm{GHz}$. The radio bursts occur during the rise of the soft X-ray flux to a weak maximum during the decay of an earlier burst. Again, a narrow, but rapid CME is observed by LASCO. The type III burst source at $164 \mathrm{MHz}$ projects onto the angular extent of the CME (Fig. A.4b). It overlies a compact brightening in the EIT image. The $237 \mathrm{MHz}$ source in the plot refers to a noise storm associated with the same active region.
The second type III group starts at $330 \mathrm{MHz}$ during the release window, as shown in the $1 \mathrm{D}$ brightness distribution in Fig. A.4a (source slightly west of central meridian). The emission is very weak. The grey bands in the $327 \mathrm{MHz}$ plot are mostly instrumental artefacts. At $164 \mathrm{MHz}$ the same bursts are seen, and are followed by the gradual start of a long-lasting broadband emission (noise storm) that shows up as the dark band after 14:30 UT in the one-dimensional intensity plots at $164 \mathrm{MHz}$ of Fig. A.4a. It is also accompanied by long-lasting microwave emission and by a new soft X-ray burst with similar time profile as the microwaves. With the exception of the short burst just after the release window the microwave emission is probably thermal. This shows again that the acceleration of type III emitting electrons occurs mainly close to the metric radio sources. The half-maximum intensity contours in Fig. A.4c show a radio source well on the disk, in association with an EUV brightening in a different active region than the first event.

While the noise storm continuum was rising at $164 \mathrm{MHz}$, a type II burst appeared in the (20-70) MHz range (Fig. A.4, 14:32-14:39 UT). It occurred well after the other signatures of electron acceleration in the corona, and well after the proton release window. There is no evidence that the coronal 

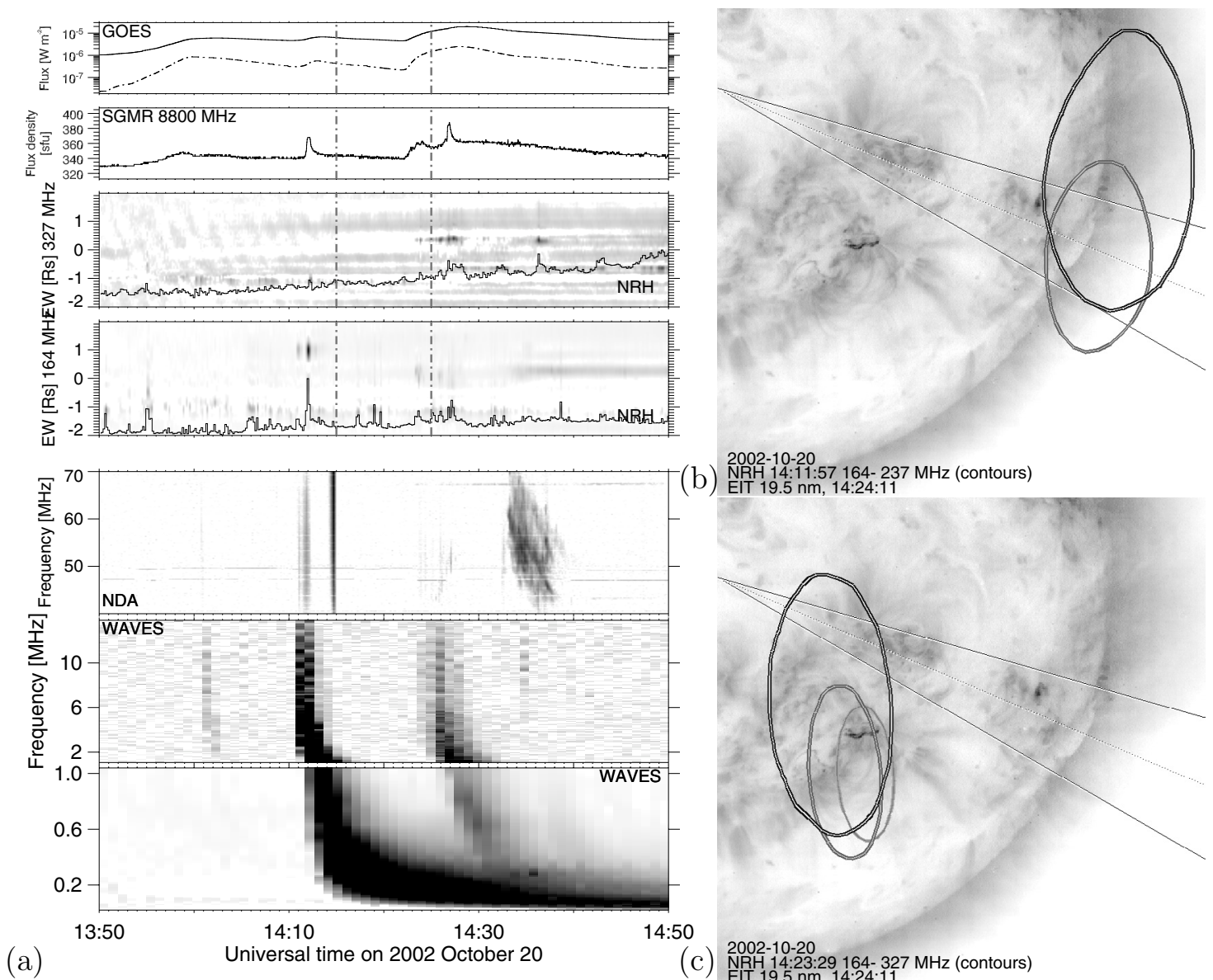

2002-10-20
(b) NRH 14:11:57 164-237 $\mathrm{MHz}$ (contours)

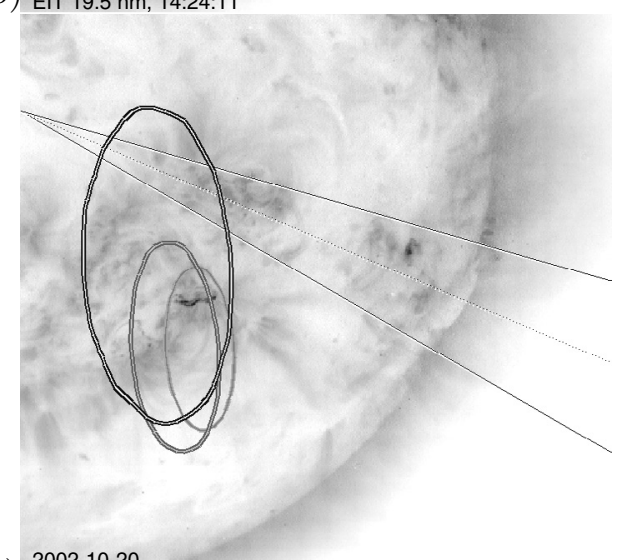

c) $2002-10-20$ N $14: 23: 29$ 164-327 MHz (contours)

Fig. A.4. 2002 October 20: a) time history of soft X-ray and radio emission; the vertical bar in the NDA spectrum near 14:15 UT, shortly after the type III burst, is a calibration signal; b) overlay of the westernmost type III radio source shortly before the release window and c) of the easternmost sources during the release window on an EIT $19.5 \mathrm{~nm}$ image. See Fig. 3 for more details.

shock revealed by this emission contributed to the early proton acceleration.

The two EUV brightenings occur in the 14:24 UT image and were not observed in the 14:12 UT image. The two active regions where they occur are connected by extended faint loops seen in the EIT image (Fig. A.4b). The nominal Earthconnected magnetic field line during this event is rooted in between the locations of the two EIT brightenings (Table 1). It therefore appears plausible that, although each of the individual active regions is far from the nominal connection to the Earth, the proton release is related to the dynamical processes proceeding in both, and that the relevant magnetic structures connect to the Earth-connected interplanetary field line at some coronal height above the metric radio sources. Wind/WAVES observed Langmuir waves during the whole day, so that we cannot ascertain the magnetic connection from the type III observations.

It may appear questionable that loop structures interconnecting two active regions in the corona are magnetically linked to the Earth at the time when the solar wind speed is as high as during the present event (Table 1). We note, however, that this observation is consistent with the recent finding (Neugebauer et al. 2002) that near solar maximum active regions may be sources of moderately fast solar wind with speeds up to more than $600 \mathrm{~km} \mathrm{~s}^{-1}$. The association of both flaring active regions with metric-to-kilometric type III bursts demonstrates the connection of both of them to open magnetic field lines. If this occurred in a streamer-like magnetic configuration, the cusp would indeed be near the Earth-connected longitude. 